\title{
Synchronization, intermittency and critical curves in a duopoly game
}

\author{
Gian-Italo Bischi ${ }^{\mathrm{a}}$, Luciano Stefanini ${ }^{\mathrm{a}}$, Laura Gardini ${ }^{\mathrm{b}}{ }^{*}$ \\ ${ }^{a}$ Istituto di Scienze Economiche, University of Urbino, 61029 Urbino, Italy \\ ${ }^{\mathrm{b}}$ Dipartimento di Metodi Quantitativi, University of Brescia, 25122 Brescia, Italy
}

Received 11 August 1997; accepted 20 October 1997

\begin{abstract}
The phenomenon of synchronization of a two-dimensional discrete dynamical system is studied for the model of an economic duopoly game, whose time evolution is obtained by the iteration of a noninvertible map of the plane. In the case of identical players the map has a symmetry property that implies the invariance of the diagonal $x_{1}=x_{2}$, so that synchronized dynamics is possible. The basic question is whether an attractor of the one-dimensional restriction of the map to the diagonal is also an attractor for the two-dimensional map, and in which sense. In this paper, a particular dynamic duopoly game is considered for which the local study of the transverse stability, in a neighborhood of the invariant submanifold in which synchronized dynamics takes place, is combined with a study of the global behavior of the map. When measure theoretic, but not topological, attractors are present on the invariant diagonal, intermittency phenomena are observed. The global behavior of the noninvertible map is investigated by studying of the critical manifolds of the map, by which a two-dimensional region is defined that gives an upper bound to the amplitude of intermittent trajectories. Global bifurcations of the basins of attraction are evidenced through contacts between critical curves and basin boundaries. (C) 1998 IMACS/Elsevier Science B.V.
\end{abstract}

Keywords: Synchronization; Riddled basins; Critical curves; Contact bifurcations; Duopoly games

\section{Introduction}

The phenomenon of synchronization of a two-dimensional discrete dynamical system

$$
T:\left(x_{1}(t), x_{2}(t)\right) \rightarrow\left(x_{1}(t+1), x_{2}(t+1)\right), t \in \mathbb{N}
$$

defined by the iteration of a map of the form $\mathbf{x}^{\prime}=T(\mathbf{x})$, where $\mathbf{x}=\left(x_{1}, x_{2}\right)$ and ' denote the unit-time advancement operator, has been in recent years the object of increasing interest in many fields. The possibility of synchronization arises when an invariant one-dimensional submanifold of $\mathbb{R}^{2}$ exists. For instance, in the case, frequently met in applications of a two-dimensional map which models the interaction of two identical one-dimensional systems, obtained by coupling two identical one-

\footnotetext{
*Corresponding author. Fax: 0039722327 655; e-mail: gardini@econ.uniurb.it
} 
dimensional maps (see $[12,9,22,14]$ ), the invariant subset on which the synchronized dynamics occurs is the diagonal

$$
\Delta=\left\{\left(x_{1}, x_{2}\right) \mid x_{1}=x_{2}\right\}
$$

In this case, the synchronized trajectories are characterized by

$$
\left(x_{1}(t), x_{2}(t)\right)=\left\{T^{t}\left(x_{1}(0), x_{2}(0)\right) \mid x_{1}(t)=x_{2}(t) \forall t \geq 0\right\}
$$

These trajectories are governed by the restriction of $T$ to the invariant submanifold on which the synchronized dynamics occur, given by the one-dimensional map

$$
f=T_{\left.\right|_{\Delta}}: \Delta \rightarrow \Delta
$$

A trajectory of $T$ starting outside of $\Delta$ is said to synchronize if

$$
\left|x_{1}(t)-x_{2}(t)\right| \rightarrow 0 \text { as } t \rightarrow \infty
$$

The basic question examined in the recent literature is whether an attractor $\mathscr{A}$ of the one-dimensional map $f$ is also an attractor located on the invariant submanifold $\Delta$, of the two-dimensional map $T$. In this context, new and interesting mathematical problems have been evidenced since measure theoretic, but not topological, attractors appear quite naturally in this context together with new striking properties of the basins (see $[2,3,20,4,15]$ ).

The very particular feature of the invariance of a submanifold of lower dimensionality than the total phase space becomes generic if the map $T$ has some symmetry property. This is often the case for maps that are obtained as models of dynamic duopoly games with identical players. The time evolution of these games, in which two players make their moves $\left(x_{1}, x_{2}\right) \in \mathscr{R}^{2}$ at discrete time periods $t=0,1,2, . .$, is often modeled by the iterations of a two-dimensional map (1). In the case of identical players, the map $T$ must remain the same under the exchange of the players, i.e. $T \circ S=S \circ T$, where $S:\left(x_{1}, x_{2}\right) \rightarrow\left(x_{2}, x_{1}\right)$ denotes the reflection through the diagonal $\Delta$. This symmetry property implies that the diagonal is mapped into itself by $T$, i.e.

$$
T(\Delta) \subseteq \Delta
$$

In such models this invariance property corresponds to the obvious statement that identical players, starting with identical initial strategies $x_{1}(0)=x_{2}(0)$, behave identically for each $t \geq 0$, even if each of them behaves chaotically. In this case, the common behavior of the two players is summarized by the dynamics of the simpler one-dimensional map (4).

In this context, it become important to see if games starting from different initial strategies of the two identical players, i.e. with initial condition outside of $\Delta$, evolve toward synchronization, so that the long-run behavior is characterized by the one-dimensional attractors of the restriction (4), included into the invariant diagonal. Of course, these attracting sets of the map $f$ are stable with respect to perturbations along $\Delta$, hence an answer to the question addressed above requires a local study of the stability with respect to perturbations transverse to $\Delta$ (or transverse stability). Moreover, when the attractors of the one-dimensional restriction (4) are also attractors for the two-dimensional map (in the usual topological sense or in a weak metric sense) the shape of the boundaries and the inner structure of their basins of attraction becomes an important question for practical purposes. The study of the basins and their qualitative changes (or bifurcations) as some parameters are let to vary requires the knowledge of the global properties of the map. 
In order to study in detail such problems a particular duopoly model is proposed in this paper, for which the restriction $f$ is conjugate to the standard logistic map. We show that the attractor $A$ of $f$ along $\Delta$ looses asymptotic stability due to the appearance of transverse unstable sets of cycles belonging to $\Delta$. The loss of asymptotic (Lyapunov) stability is in general associated with one of the following different types of global dynamics determined by the different behavior of the local unstable manifolds of the transversally repelling cycles: the unstable sets can (i) be folded back towards $\Delta$, thus giving intermittency phenomena, or (ii) may belong to the basin of another attractor, in which case the phenomenon of riddled basins can be obtained (see $[2,20]$ ).

As noticed in $[20,3,4,14]$, the difference between these two situations is determined by the global properties of the map. On the contrary, the study of the Lyapunov exponents, which is the method usually followed to study the onset of transverse instability, only give local informations in a neighborhood of $\Delta$. This paper is organized as follows:

In Section 2, the economic system modeled by the dynamic duopoly game is briefly described, and the general properties of the noninvertible two-dimensional map, whose iteration gives the time evolution of the duopoly game, are studied in the symmetric case of identical players. The basic definitions concerning attractors, basins and critical curves are recalled, and the main properties of the attractors of the synchronized trajectories are described. A method to obtain the boundary separating the basin of attraction of infinity from the basin of bounded trajectories is presented.

In Section 3, the critical curves of the map are used to define the boundaries of an invariant area of the phase plane inside which the bounded trajectories are confined when the invariant set on the diagonal looses transverse stability.

In Section 4, some global bifurcations are described: one that changes the structure of the basins, one that causes the disappearance of the invariant area described in Section 3 and one causing the disappearance of any bounded attractor. Such global bifurcations are characterized as contact bifurcations, related to tangencies between critical curves and basin boundaries.

The main purpose of this work is that of stressing the crucial role of the critical curves (see $[11,18]$ ) in the characterization of the global properties of the map considered in this paper, and, in particular, their role in the occurrence of the different dynamical behaviors described above.

\section{General properties of the duopoly model}

\subsection{The economic model}

We consider a repeated Cournot duopoly game which describes a market where two quantity-setting firms, producing homogeneous goods, update their production strategies at discrete time periods $t=0,1,2, .$. The dynamic game is based on the assumption that the two producers have not a complete knowledge of the market, hence they behave adaptively, following a bounded rationality adjustment process based on a local estimate of the marginal profit $\partial \Pi_{i} / \partial x_{i}$ (see [5] and references therein). At each time period a firm decides to increase its production $x_{i}$ if it perceives positive marginal profit, or decreases its production if the marginal profit is negative

$$
x_{i}(t+1)=x_{i}(t)+v_{i} x_{i}(t) \frac{\partial \Pi_{i}\left(x_{1}, x_{2}\right)}{\partial x_{i}}, \quad i=1,2 ; \quad t=0,1,2, \ldots,
$$

where $\Pi_{i}\left(x_{1}, x_{2}\right)$ is the one-period profit of producer $i$ and $v_{i}$ is a positive parameter which represents the 
relative speed of production adjustment of producer $i$. As usual in duopoly models, the price of the good is determined by the total supply $Q(t)=x_{1}(t)+x_{2}(t)$ through a given inverse demand function $p=g(Q)$, so that the one-period profit for firm $i$ is given by

$$
\Pi_{i}\left(x_{1}, x_{2}\right)=x_{i} g\left(x_{1}+x_{2}\right)-c_{i} x_{i}, \quad i=1,2
$$

where the positive constants $c_{i}$ represent the marginal costs of the two firms. With this assumption the time evolution of the dynamic game (5) is determined by the iteration of the following two-dimensional map:

$$
T:\left\{\begin{array}{l}
x_{1}^{\prime}=x_{1}+v_{1} x_{1}\left[g\left(x_{1}+x_{2}\right)+x_{1} \frac{\partial g}{\partial x_{1}}-c_{1}\right] \\
x_{2}^{\prime}=x_{2}+v_{2} x_{2}\left[g\left(x_{1}+x_{2}\right)+x_{2} \frac{\partial g}{\partial x_{2}}-c_{2}\right]
\end{array}\right.
$$

Starting from some nonnegative initial productions

$$
\left(x_{1}(0), x_{2}(0)\right)=\left(x_{1,0}, x_{2,0}\right)
$$

the iteration of (7) uniquely defines the forward time evolution of the repeated game, represented by the trajectory $\left(x_{1}(t), x_{2}(t)\right)=T^{t}\left(x_{1,0}, x_{2,0}\right), t \geq 0$.

In this model, the producer labelled by $i$ is characterized by the two parameters $v_{i}$ and $c_{i}$, representing the relative speed of adjustment and the marginal cost, respectively. In the case of identical producers characterized by the same values of the parameters

$$
c_{1}=c_{2}=c \text { and } v_{1}=v_{2}=v
$$

the map has a symmetry property: it remains the same after a reflection through the diagonal $\Delta$ of equation $x_{1}=x_{2}$, obtained by the operator $P:\left(x_{1}, x_{2}\right) \rightarrow\left(x_{2}, x_{1}\right)$. This implies that the diagonal $\Delta$ is an invariant line for the map $T$, so that synchronized dynamics can occur on $\Delta$.

In the following, we consider a particular duopoly game (7) obtained by taking a linear demand function, very often used in economic modeling, given by $p=g(Q)=a-b\left(x_{1}+x_{2}\right)$, with $a, b$ positive constants. With this choice, the map (7) becomes

$$
T:\left\{\begin{array}{l}
x_{1}^{\prime}=x_{1}\left[1+v_{1}\left(a-c_{1}\right)-2 b v_{1} x_{1}-b v_{1} x_{2}\right] \\
x_{2}^{\prime}=x_{2}\left[1+v_{2}\left(a-c_{2}\right)-2 b v_{2} x_{2}-b v_{2} x_{1}\right]
\end{array}\right.
$$

\subsection{Invariant sets and local stability properties}

The map (10) is a noninvertible map of the plane, i.e., starting from some nonnegative initial production strategy (8), the iteration of (10) uniquely defines the forward trajectory, whereas the backward iteration of (10) is not uniquely defined because a point $\left(x_{1}{ }^{\prime}, x_{2}{ }^{\prime}\right)$ of the plane may have several preimages, obtained by solving the fourth degree algebraic system (10) with respect to $x_{1}$ and $x_{2}$ (see [18] for a description of the main properties of noninvertible maps of the plane).

The map (10) is characterized by the presence of four fixed points: $E_{0}=(0,0), E_{1}=\left(a-c_{1} / 2 b, 0\right)$, $E_{2}=\left(0, a-c_{2} / 2 b\right)$, located on the invariant coordinate axes, and

$$
E_{*}=\left(\frac{a+c_{2}-2 c_{1}}{3 b}, \frac{a+c_{1}-2 c_{2}}{3 b}\right)
$$


which is interior to the positive quadrant provided that

$$
2 c_{1}<a+c_{2} \text { and } 2 c_{2}<a+c_{1}
$$

It is easy to verify that when (12) is satisfied, the fixed point $E_{*}$ represents the unique Nash equilibrium for the duopoly game.

An important feature of the map (10) is that it always has an asymptotically stable attractor at infinity, i.e. it can generate unbounded trajectories, if the initial condition (8) is taken sufficiently far from the origin, i.e., in a suitable neighborhood of infinity. In fact, if $\left\|\left(x_{1,0}, x_{2,0}\right)\right\|$ is sufficiently large then the first iterate of (7) gives negative values $x_{i}^{\prime}<0, i=1,2$, so that the successive iterates give negative and decreasing values. This implies that any attractor at finite distance cannot be globally attracting in $\mathbb{R}_{+}^{2}$.

It is worth noticing that each coordinate axis $x_{j}=0, j=1,2$, is invariant for the map (10) since $x_{j}=0$ gives $x_{j}^{\prime}=0$. The dynamics on the invariant manifold $x_{j}=0$ is governed by the restriction of the map $T$ to that axis, given by the following one-dimensional map, obtained from (7) with $x_{j}=0$.

$$
x_{i}^{\prime}=f_{i}\left(x_{i}\right)=\left(1+v_{i}\left(a-c_{i}\right)\right) x_{i}-2 b v_{i} x_{i}^{2} \quad i=1,2
$$

The two restrictions $f_{1}$ and $f_{2}$ are conjugate to the standard logistic map

$$
z^{\prime}=\mu_{i} z(1-z), \quad i=1,2
$$

through the linear transformation

$$
x_{i}=\frac{1+v_{i}\left(a-c_{i}\right)}{2 b v_{i}} z, \quad i=1,2
$$

from which we obtain the relation

$$
\mu_{i}=1+v_{i}\left(a-c_{i}\right), \quad i=1,2
$$

In the symmetric case (9) of identical producers, the two restrictions of $T_{s}$ to the invariant coordinate axes are given by the same map $f_{1}=f_{2}$. Furthermore, in this case, as stated above, also the diagonal $x_{1}=x_{2}$ is invariant. Under the assumption (9) the map (10) becomes

$$
T_{s}:\left\{\begin{array}{l}
x_{1}^{\prime}=(1+v(a-c)) x_{1}-2 b v x_{1}^{2}-b v x_{1} x_{2} \\
x_{2}^{\prime}=(1+v(a-c)) x_{2}-2 b v x_{2}^{2}-b v x_{1} x_{2}
\end{array}\right.
$$

the Nash equilibrium (11) becomes

$$
E_{*}=\left(\frac{a-c}{3 b}, \frac{a-c}{3 b}\right) \in \Delta
$$

and the two boundary equilibria $E_{1}$ and $E_{2}$ are in symmetrical positions with respect to the line $\Delta$. The restriction $\left.T_{s}\right|_{\Delta}$ of $T_{s}$ to $\Delta$ is given by

$$
x^{\prime}=f(x)=(1+v(a-c)) x-3 b v x^{2}
$$

which is conjugate to the standard logistic map

$$
z^{\prime}=\mu z(1-z)
$$


with parameter

$$
\mu=1+v(a-c)
$$

by the linear transformation

$$
x=\frac{1+v(a-c)}{3 b v} z
$$

Thus, the dynamical behavior of the restriction of $T$ to the invariant manifold $\Delta$, where synchronized dynamics of the identical players takes place, can be obtained from the well known behavior of the standard logistic map by an homeomorphism.

We shortly recall some properties of the logistic map (20) to be used below. The positive fixed point $z^{*}=\mu-1 / \mu$ is stable for $1<\mu<3$, corresponding to $0<v(a-c)<2$ for the map (19), and its basin of attraction is given by $D_{z}=(0,1)$, corresponding to $D_{x}=(0,(1+v(a-c)) / 3 b v)$ for the map in (19). Of course, the positive fixed point of (19) on the diagonal $\Delta$ coincides with the Nash equilibrium (18) of the duopoly game. At $\mu=\mu_{0}=3$, the fixed point $z^{*}$ looses stability through a flip bifurcation, and for $3<\mu<1+\sqrt{6}$ an attracting cycle of period two is the only attractor of (20). Also, this cycle undergoes a flip bifurcation at $\mu=\mu_{1}=1+\sqrt{6}$ that creates an attracting cycle of period four, and so on, a sequence of flip bifurcations occur at $\mu=\mu_{n}, n \in \mathbb{N}$, leads to chaotic behavior (see e.g. [7,19]). The sequence $\left\{\mu_{n}\right\}$ is increasing and convergent to $\mu^{*}=3.5699 \ldots$ (called Myrberg, or Feigenbaum, point). For $\mu>\mu^{*}$ the $\omega$-limit set of the generic trajectory of the logistic map starting with $z_{0} \in(0,1)$ is either an attracting cycle or cyclic-invariant chaotic intervals or a weak attractor represented by a Cantor set (see e.g. [19,23]).

In any case, for each $\mu \in(2,4)$, the existing attractor belongs to the absorbing interval, $I=\left[\mathfrak{C}_{1}, \mathfrak{C}\right]$, which is an attracting set bounded by two critical points, $\mathfrak{C}=f\left(\mathfrak{C}_{-1}\right)=\mu / 4, \mathfrak{C}_{1}=f^{2}\left(\mathfrak{C}_{-1}\right)=(\mu / 4$ $(1-\mu / 4)), \mathfrak{C}_{-1}=1 / 2$ being the point of maximum of $f$. Absorbing or trapping means that any point in a suitable neighborhood of $I$ has the $\omega$-limit set in $I$. More precisely, for the standard logistic map (20) we have that any point inside the interval $[0,1]$ has the $\omega$-limit set in $I$. For the synchronized trajectories of $T$, occurring on $\Delta$, this means that for each $v(a-c) \in(1,3)$, any trajectory starting from a point $\left(x_{0}, x_{0}\right) \in \Delta$, with $x_{0} \in(0,(1+v(a-c)) / 3 b v)$, has its $\omega$-limit set inside the segment of diagonal $I_{\Delta}=C_{1} C \subset \Delta$, where $C=\left((1+v(a-c))^{2} / 12 b v,(1+v(a-c))^{2} / 12 b v\right)$ is the rank-1 critical point (the maximum value) of $\left.T\right|_{\Delta}$, and $C_{1}=T_{s}(C)$.

The point $\mu^{*}$ is also the limit point for the decreasing sequence $\left\{\bar{\mu}_{n}\right\}$ of parameter's values at which homoclinic bifurcations of the cycles of period $2^{n}$ occur. At each $\bar{\mu}_{n}$ the logistic map is chaotic, with absolutely continuous invariant measure on a chaotic attractor given by the union of $2^{n+1}$ subintervals inside $I$. Analogous sequences of homoclinic bifurcations of cycles of period different from $2^{n}$ occur: indeed, for $\mu \in\left(\mu^{*}, 4\right)$ there are infinitely many 'windows' (following [7]) or 'boxes' (following [19]) started by fold bifurcations, followed by period doubling cascades and closed by homoclinic bifurcations. So, infinitely many values of $\bar{\mu}$ exist at which homoclinic bifurcations occur. At each of such values of $\mu$, the logistic map has cyclic chaotic intervals with absolutely continuous invariant measure. For $\mu>4$, corresponding to $v(a-c)>3$ in (19), the generic trajectory of the logistic map is divergent (see e.g. [8]).

Thus, for $2<v(a-c)<3$ there are infinitely many parameters' sets at which the two-dimensional map $T_{s}$ has synchronized chaotic dynamics on the invariant submanifold $\Delta$. 
In the following, we shall denote by $\mathscr{A}$ the attractor of $f=\left.T_{s}\right|_{\Delta}$. It belongs to the segment $I_{\Delta}=C C_{1} \subset \Delta$. This segment is absorbing with respect to perturbations along $\Delta$, but in order to see if it is a trapping segment for the two-dimensional map $T_{s}$ its transverse attractivity must be examined.

In order to study the transverse stability of the attractor $\mathscr{A} \subseteq I_{\Delta}$ we consider the Jacobian matrix of the map (17),

$$
D T_{s}\left(x_{1}, x_{2}\right)=\left[\begin{array}{cc}
1+v\left(a-c-4 b x_{1}-b x_{2}\right) & -v b x_{1} \\
-v b x_{2} & 1+v\left(a-c-b x_{1}-4 b x_{2}\right)
\end{array}\right]
$$

that computed on the line $\Delta$ assumes the structure

$$
D T_{s}(x, x)=\left[\begin{array}{ll}
l(x) & m(x) \\
m(x) & l(x)
\end{array}\right]
$$

with $l(x)=1+v(a-c)-5 v b x$ and $m(x)=-v b x$. The eigenvalues are

$$
\begin{aligned}
& \lambda_{\|}=l(x)+m(x)=1+v(a-c)-6 v b x, \quad \text { with eigen vector } \mathbf{r}_{\|}=(1,1), \\
& \lambda_{\perp}=l(x)-m(x)=1+v(a-c)-4 v b x, \quad \text { with eigen vector } \mathbf{r}_{\perp}=(1,-1)
\end{aligned}
$$

Of course, the eigenvalue $\lambda_{\|}$, associated with the invariant manifold along the line $\Delta$, coincides with the multiplier of the restriction $\left.T_{s}\right|_{\Delta}$ given by (19). Notice also that the eigenvector associated with the other eigenvalue is always orthogonal to $\Delta$ and independent of $x$.

For a $k$-cycle $\left\{\left(\alpha_{1}, \alpha_{1}\right), \ldots,\left(\alpha_{k}, \alpha_{k}\right)\right\}$ of (17), embedded into the invariant line $\Delta$ where synchronized dynamics take place and corresponding to the cycle $\left\{\alpha_{1}, \ldots, \alpha_{k}\right\}$ of the one-dimensional quadratic map (19), the two multipliers are

$$
\begin{aligned}
& \lambda_{\|}^{(k)}=\prod_{i=1}^{k}\left(l\left(\alpha_{i}\right)+m\left(\alpha_{i}\right)\right)=\prod_{i=1}^{k}\left(1+v(a-c)-6 b v \alpha_{i}\right) \\
& \lambda_{\perp}^{(k)}=\prod_{i=1}^{k}\left(l\left(\alpha_{i}\right)-m\left(\alpha_{i}\right)\right)=\prod_{i=1}^{k}\left(1+v(a-c)-4 b v \alpha_{i}\right)
\end{aligned}
$$

Also for the cycles the conditions for stability and local bifurcations along $\Delta$ are the same as for the corresponding cycle of the quadratic map (19). Hence, in the following we focus our attention on the transverse stability of the invariant sets located on the line $\Delta$. For the fixed point $E_{*}$ the transverse eigenvalue is

$$
\lambda_{\perp}^{\left(E_{*}\right)}=1-\frac{1}{3} v(a-c)
$$

so it is transversally attracting for all parameters that give bounded dynamics on $\Delta$, i.e. for $0<v(a-c)<3$. For $0<v(a-c)<2, E_{*}$ is an asymptotically stable node, whereas it is a saddle for $2<v(a-c)<6$, with unstable set along $\Delta$ and local stable set orthogonal to it. Notice that at $v(a-c)=3$, we have $\lambda_{\perp}^{\left(E_{*}\right)}=0$.

For sufficiently small values of $v(a-c)$ any attractor of the restriction $\left.T_{s}\right|_{\Delta}$ is also an asymptotically stable attractor also for the two-dimensional map $T$.

Before considering more complex situations we recall some definitions. Let $\mathscr{A}$ be a closed invariant set such that $T(\mathscr{A}) \equiv \mathscr{A}$.

Definition 1. The stable set of $\mathscr{A}$, denoted by $\mathscr{B}(\mathscr{A})$, is the set of points $x$ for which $\omega(x) \subseteq \mathscr{A}$, i.e. the $\omega$-limit sets of $x$ belongs to $\mathscr{A}$. 
Note that when $T$ is a noninvertible map any cycle has a stable set, even a repelling node or a repelling focus.

Definition 2. $\mathscr{A}$ is an asymptotically stable attractor (or topological attractor) if it is Lyapunov stable, i.e. for every neighborhood $U$ of $\mathscr{A}$ there exists a neighborhood $\operatorname{Vof} \mathscr{A}$ such that $T^{t}(V) \subset U \forall t \geq 0$, and $\mathscr{B}(\mathscr{A})$ contains a neighborhood of $\mathscr{A}$.

If $\mathscr{A}$ is a topological attractor then a neighborhood $W \supset \mathscr{A}$ exists such that $T^{t}(x) \rightarrow \mathscr{A}$ as $t \rightarrow+\infty$ for any $x \in W$. In this case, the stable set $\mathscr{B}(\mathscr{A})$, also called basin of attraction, is given by $\mathscr{B}(\mathscr{A})=\bigcup_{t \geq 0} T^{-t}(W)$.

An attractor $\mathscr{A}$ of the one-dimensional map (19) is also an asymptotically stable attractor of the twodimensional symmetric map $T_{s}$ if and only if all the trajectories belonging to $\mathscr{A}$ are transversally attracting. If $\mathscr{A}$ is a $k$-cycle this is ensured by the condition $\left|\lambda_{\perp}^{(k)}\right|<1$, where $\lambda_{\perp}^{(k)}$ is given by (25), whereas if $\mathscr{A}$ is a chaotic attractor a stability condition can be given in terms of the natural transverse Lyapunov exponent

$$
L_{\perp}=\lim _{\tau \rightarrow \infty} \frac{1}{\tau} \sum_{t=0}^{\tau} \ln |1+v(a-c)-4 b v x(t)|
$$

where $x(0) \in \mathscr{A}$ and $\{x(t)\}$ is the corresponding trajectory generated by the map $f=\left.T_{s}\right|_{\Delta}$. If $L_{\perp}<0$ for each trajectory starting inside $\mathscr{A}$ then $\mathscr{A}$ is asymptotically stable.

Of course, if $x(0)$ is a point of a $k$-cycle, or belonging to the stable set of a $k$-cycle, then $L_{\perp}=\ln \left|\lambda_{\perp}^{(k)}\right|$, so in this case the condition $L_{\perp}<0$ is equivalent to $\left|\lambda_{\perp}^{(k)}\right|<1$. Instead, if $\mathscr{A}$ is a chaotic attractor, with absolutely continuous invariant measure, then almost all the aperiodic trajectories in $\mathscr{A}$ give the same value of $L_{\perp}$. It is clear that in this case it is difficult to prove that $\mathscr{A}$ is a topological attractor (since we should check the values of $\left|\lambda_{\perp}^{(k)}\right|$ for all the cycles embedded in it), whereas it is easier to see when $\mathscr{A}$ is not a topological attractor. In fact, for this it is enough to find at least one $k$-cycle embedded in $\mathscr{A}$ with $\left|\lambda_{\perp}^{(k)}\right|>1$. In this case, the fact that $L_{\perp}<0$ for the generic aperiodic trajectory in $\mathscr{A}$ means that $\mathscr{A}$ is transversally attracting on the average, in the sense that the majority of the trajectories on $\mathscr{A}$ are transversally attracting, but some (even infinitely many) trajectories inside $\mathscr{A}$ can exist whose transverse Lyapunov exponent is positive. In other words, transversally repelling trajectories can be embedded into a chaotic set which is attracting only 'on average'. This type of stability is known as weak stability or stability in Milnor sense (from [16], see also [2,4]).

Definition 3. A closed invariant set $\mathscr{A}$ is said to be a weak attractor in Milnor sense (or simply Milnor attractor) if its stable set $\mathscr{B}(\mathscr{A})$ has positive Lebesgue measure.

Note that a topological attractor is also a Milnor attractor, whereas the converse is not true. Really the more general notion of Milnor attractor has been introduced to evidence the existence of invariant sets which 'attract' many points even if they are not attractors in the usual topological sense. In this case, in [16] $\mathscr{B}(\mathscr{A})$ is called 'realm of attraction', reserving the term 'basin' when $\mathscr{B}(\mathscr{A})$ is an open set. However, since the term basin is more standard in the literature, we shall use such term even when $\mathscr{A}$ is a Milnor (but not topological) attractor, for which $\mathscr{B}(\mathscr{A})$ is not, in general, an open set. 
If $\mathscr{A} \subset \Delta$ is a chaotic attractor of $\left.T_{s}\right|_{\Delta}$ with absolutely continuous invariant measure, then the condition $L_{\perp}<0$ ensures that, for any neighborhood $W$ of $\mathscr{A}, \mu(\mathscr{B}(\mathscr{A}) \cap W)>0$, where $\mu$ denotes Lebesgue measure. Thus, a sufficient condition for a chaotic attractor of $\left.T_{s}\right|_{\Delta}$ (with absolutely continuous invariant measure) to be a Milnor, but not topological, attractor for the two-dimensional map $T_{s}$, is that

(a) at least one $k$-cycle embedded in $\mathscr{A}$ is transversally repelling, i.e. $\left|\lambda_{\perp}^{(k)}\right|>1$, and

(b) the Lyapunov exponent (27) computed for the generic aperiodic trajectory is negative.

In other words, the transition (or bifurcation) of an invariant set $\mathscr{A} \subset \Delta$ from topological attractor of $T_{s}$ to a Milnor (but not topological) one occurs when the eigenvalue $\lambda_{\perp}^{(k)}$ of one (or more) cycle embedded in $\mathscr{A}$ exits the unit circle while all the other cycles in $\mathscr{A}$ are transversally stable. We remark that 'generally' this occurs for a cycle of low period. This fact has been noticed in [13], qualitative reasons to explain this are given in [15], and it seems to be confirmed in our example.

We note that in the existing literature, the studies on Milnor attractors, riddled basins and synchronization phenomena are often given for the study of coupled maps, i.e. maps of type

$$
\left(x^{\prime}, y^{\prime}\right)=F(x, y)=\left(f_{a}(x)+\varepsilon g(x-y), f_{a}(y)+\varepsilon g(y-x)\right)
$$

so that the restriction $\left.F\right|_{\Delta}=f_{a}$ is independent of the coupling parameter $\varepsilon$ (see $\left.[12,9,22,14,15]\right)$. In this case, the main bifurcations of the invariant sets of the two-dimensional map belonging to the subspace where synchronized dynamics occur, such as the bifurcation from topological attractor to a non topological one, can be studied as a function of the coupling parameter $\varepsilon$ without altering the dynamical properties of the attractors of the restriction, depending only on the parameter $a$. This kind of study is not possible in our example. In fact the parameter $v$, that we are changing as a bifurcation parameter in order to study the transverse stability along $\Delta$, influences both the coupling and the dynamics of the restriction $\left.T_{s}\right|_{\Delta}$. The same is true for the other parameters of the map (17).

A numerical computation of $L_{\perp}$, performed with $a=10, b=0.5$ and $c=3$, is shown in Fig. 1 as a function of the parameter $v^{1}$. With this set of parameters, a numerical computation of the cycles on $\Delta$ up to period 16, together with their transverse eigenvalues, has been performed with different values of the parameter $v$. We have obtained that the conditions for asymptotic stability of the attractor $\mathscr{A}$ along $\Delta$ are fulfilled for $v<0.37562$ (we remark that for this set of parameters the Feigenbaum point is given by $\left.v^{*}=0.36713 \ldots\right)$.

\subsection{Basins of attraction}

The basin of attraction $\mathscr{B}(\mathscr{A})$ of the bounded attractor existing on $\Delta$ belongs to the complementary set of the basin of infinity $\mathscr{B}(\infty)$, defined as the set of points which generate unbounded trajectories. If no other bounded attractor (or attractor at finite distance) exists, then $\mathscr{B}(\mathscr{A})$ is given by the set of the interior points of the set $\mathbb{R}^{2} / \mathscr{B}(\infty)$.

Let $\partial \mathscr{B}(\infty)$ be the boundary of $\mathscr{B}(\infty)$, which is also the boundary of $\mathscr{B}(\mathscr{A})$, when $\mathscr{A}$ is the only attractor at finite distance. It behaves as a repelling set for the points near it, since it acts as a watershed

\footnotetext{
${ }^{1}$ The results obtained with these fixed values of the parameters $a, b$ and $c$ are general in the sense that different values give similar bifurcations. The values chosen to perform the numerical simulations are such that the Nash equilibrium $E_{*}$ is always positive.
} 


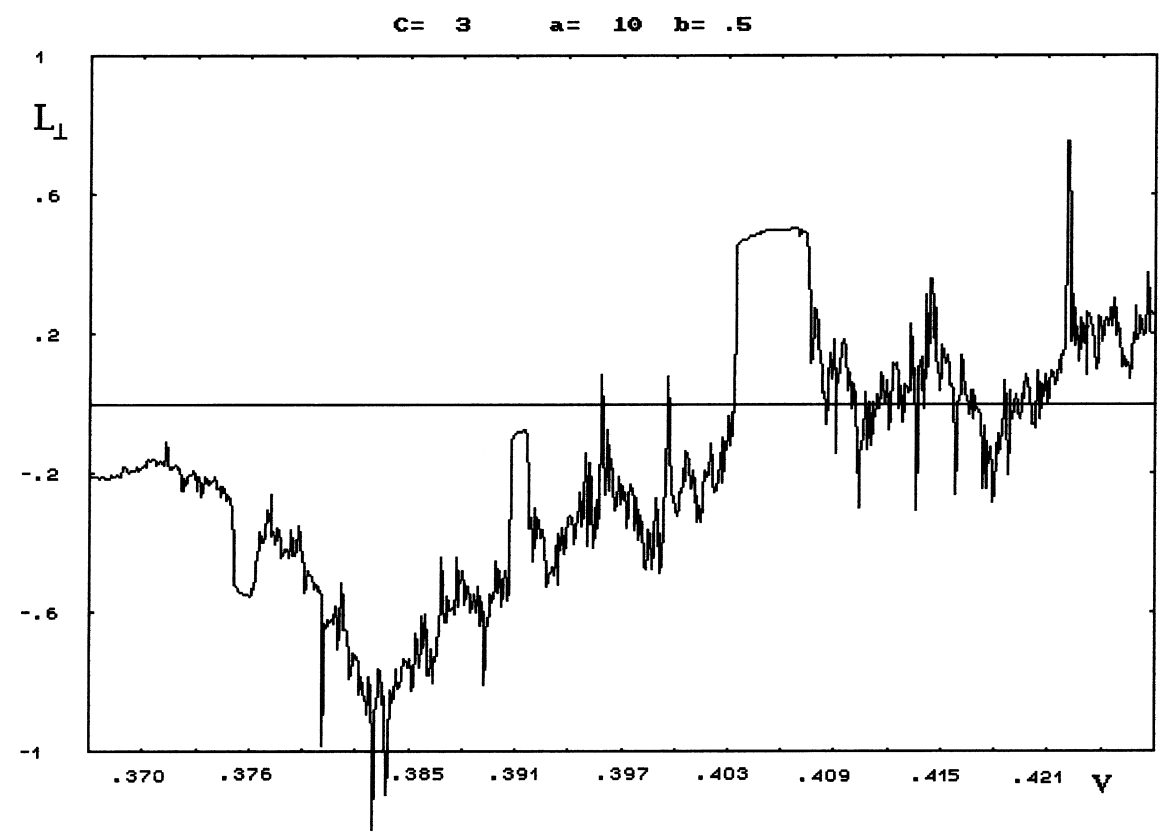

Fig. 1. Natural transverse Lyapunov exponent $L_{\perp}$ as a function of $v$ ranging from 0.367 to 0.427 , with $c=3, a=10, b=0.5$. Each point is obtained by iterating the map (starting from an initial condition on the diagonal) 10000 times to eliminate transient behavior, and then averaging over another 50000 iterations.

for the trajectories of the map $T$. Points belonging to $\partial \mathscr{B}(\infty)$ are mapped into $\partial \mathscr{B}(\infty)$ both under forward and backward iteration of $T_{s}$. More precisely, it is invariant for application of $T_{s}^{-1}$ and mapped into itself by $T_{s}$, i.e., $T_{s}^{-1}(\partial B(\infty))=\partial B(\infty)$ and $T_{s}(\partial \mathscr{B}(\infty)) \subseteq \partial \mathscr{B}(\infty)$ (see [17,18]). This implies that if a saddle-point, or a saddle-cycle, belongs to $\partial \mathscr{B}(\infty)$, then $\partial \mathscr{B}(\infty)$ must also contain the whole stable manifold $W^{s}$, and if a repelling cycle (node or focus) belongs to it then $\partial \mathscr{B}(\infty)$ contains all the preimages of the cycle (see $[11,18]$ ).

Let us first consider the dynamics of $T$ restricted to the invariant axes. From the one-dimensional restrictions $f_{i}\left(x_{i}\right), i=1,2$, defined in (13), we can easily deduce that bounded trajectories along the invariant axis $x_{j}=0, j \neq i$, are obtained, if $v_{i}\left(a-c_{i}\right)<3$, provided that the initial conditions are taken inside the segment $\omega_{i}=\left[0,0_{-1}^{(i)}\right]$, where $0_{-1}^{(i)}$ is the rank-1 preimage of the origin computed according to the restriction $f_{i}$, i.e.

$$
0_{-1}^{(i)}=\frac{1+v_{i}\left(a-c_{i}\right)}{2 b v_{i}}, \quad i=1,2
$$

Instead, negatively divergent trajectories along the invariant axis $x_{j}=0$ are obtained starting from an initial condition out of the segment $\omega_{i}$. Of course, under the assumption of identical producers (idem) the two segments are congruent and symmetric with respect to $\Delta$ being $0_{-1}^{(1)}=0_{-1}^{(2)}=$ $(1+v(a-c)) / 2 b v$.

The segments $\omega_{1}$ and $\omega_{2}$ on the two coordinate axes play an important role in the determination of $\partial \mathscr{B}(\infty)$ for the two-dimensional map $T_{s}$. In fact 
(a) from the computation of the eigenvalues of the cycles belonging to $\omega_{1}$ and $\omega_{2}$ we have that the direction transverse to the coordinate axes is always repelling, and

(b) point $\left(x_{1,0}, x_{2,0}\right)$ generates a divergent trajectory if $x_{1,0}<0$ or $x_{2,0}<0$. From (a) and (b) it follows that $\omega_{1}$ and $\omega_{2}$ belong to $\partial \mathscr{B}(\infty)$, as well as their preimages of any rank. From these arguments the following proposition can be stated, that gives an exact delimitation of $\partial \mathscr{B}(\infty)$.

Proposition 1. Let $1<v(a-c)<3$ and $\omega_{1}=\left[0,0_{-1}^{(1)}\right], \omega_{2}=\left[0,0_{-1}^{(2)}\right]$ be the segments of the coordinate axes $x_{1}$ and $x_{2}$, respectively, with $0_{-1}^{(i)}, i=1,2$ defined in (29). Then

$$
\partial \mathscr{B}(\infty)=\left(\bigcup_{n=0}^{\infty} T_{s}^{-n}\left(\omega_{1}\right)\right) \cup\left(\bigcup_{n=0}^{\infty} T_{s}^{-n}\left(\omega_{2}\right)\right)
$$

In fact, since $\omega_{1}$ and $\omega_{2}$ belong to $\partial \mathscr{B}(\infty)$ also their preimages of any rank belong to $\partial \mathscr{B}(\infty)$. In order to show that, for the map $T_{s}$, the whole $\partial \mathscr{B}(\infty)$ is given by the union of such preimages we compute $\omega_{1}^{-1}=T_{s}^{-1}\left(\omega_{1}\right)$ and $\omega_{2}^{-1}=T_{s}^{-1}\left(\omega_{2}\right)$. Let us consider a generic point $P=(0, p) \in \omega_{2}$, i.e. $0<p<(1+v(a-c)) / 2 b v$. Its preimages are the real solutions of the algebraic system obtained from (map) with $\left(x_{1}^{\prime}, x_{2}^{\prime}\right)=(0, p)$

$$
\left\{\begin{array}{l}
x_{1}\left[1+v(a-c)-2 b v x_{1}-b v x_{2}\right]=0 \\
(1+v(a-c)) x_{2}-2 b v x_{2}^{2}-b v x_{1} x_{2}=p
\end{array}\right.
$$

From the first of (31), we obtain $x_{1}=0$ or

$$
1+v(a-c)-2 b v x_{1}-b v x_{2}=0,
$$

which means that if the point $P$ has preimages, then they must be located either on the same invariant axis or on the line of Eq. (32). With $x_{1}=0$, the second equation in (31) becomes a second degree algebraic equation which has two distinct, coincident or no real solutions. A similar conclusion holds if (32) is used to eliminate a state variable in the second equation of (31). From this, we can deduce that the generic point $P$ of $\omega_{2}$ can have no preimages or two preimages on the same axis (which are the same obtained by the restriction of $T_{s}$ to the axis $x_{2}$ ) or four preimages, two on the same axis and two on the line of Eq. (32). This implies that the points of $\omega_{2}^{-1}$ are located on the same axis and on the line (32).

Following the same procedure we can state that $\omega_{2}^{-1}$ has preimages on itself and on the line of equation

$$
1+v(a-c)-b v x_{1}-2 b v x_{2}=0
$$

It is straightforward to see that the origin $O=(0,0)$ has always 4 preimages:

$$
\begin{aligned}
& O_{-1}^{(0)}=(0,0) ; O_{-1}^{(1)}=\left(\frac{1+v(a-c)}{2 b v}, 0\right) ; O_{-1}^{(2)}=\left(0, \frac{1+v(a-c)}{2 b v}\right) \text { and } \\
& O_{-1}^{(3)}=\left(\frac{1+v(a-c)}{3 v b}, \frac{1+v(a-c)}{3 v b}\right)
\end{aligned}
$$

where $O_{-1}^{(3)}$ is located on the intersection of the lines (32) and (33). Notice that $(1+v(a-c)) / 3 v b=f^{-1}(0)$, i.e. $O_{-1}^{(3)}$ is the rank-1 preimage of the origin computed by the restriction of $T_{s}$ on the diagonal $\Delta$. It turns out that $T_{s}^{-1}\left(\omega_{1} \cup \omega_{2}\right)$ is made up of four segments that form the 


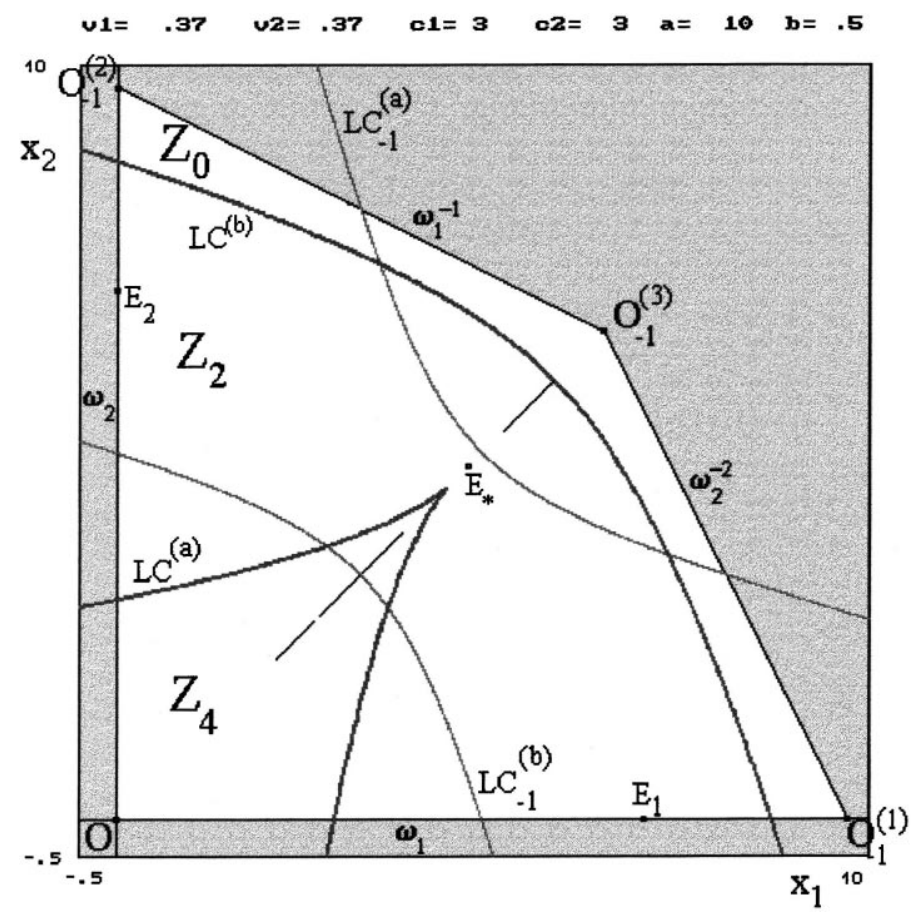

Fig. 2. For parameters' values $c=3, a=10, b=0.5$ (as in Fig. 1) and $v=0.37$ the quadrilateral $O O_{-1}^{(1)} O_{-1}^{(3)} O_{-1}^{(2)}$, which is the boundary of $\mathscr{B}(\infty)$ for this set of parameters is represented. The grey points generate unbounded trajectories, whereas a generic point taken in the white region generates a trajectory converging to the one-dimensional attractor $\mathscr{A}$ (represented by black points) embedded into the diagonal. The regions $Z_{4}, Z_{2}$ and $Z_{0}$, whose points have four, two or no preimages respectively, are bounded by the critical curves $L C^{(\mathrm{a})}$ and $L C^{(\mathrm{b})}$, as explained in Section 2.4. The branches of the critical curves $L C^{-1}$ and $L C$ are represented by thin and thick lines, respectively.

quadrilateral $O O_{-1}^{(1)} O_{-1}^{(3)} O_{-1}^{(2)}$ (see Fig. 2). All the points outside it generate divergent trajectories, i.e., the complementary of the quadrilateral $O O_{-1}^{(1)} O_{-1}^{(3)} O_{-1}^{(2)}$ constitutes the immediate basin of infinity. In fact, the initial conditions (8) with $x_{1,0}<0$, as well those with $x_{2,0}<0$, generate divergent trajectories. Furthermore, initial conditions on the right of $\omega_{2}^{-1}$ give $x_{1}^{\prime}<0$ and those above $\omega_{2}^{-1}$ give $x_{2}^{\prime}<0$. This completes the proof of the Proposition 1, since the whole basin boundary $\partial \mathscr{B}(\infty)$ is given by the boundary of the immediate basin and its preimages (if any).

In the situation shown in Fig. 2 (and in Fig. 3 as well) the quadrilateral $O O_{-1}^{(1)} O_{-1}^{(3)} O_{-1}^{(2)}$, obtained by $\omega_{1}, \omega_{2}$ and its rank-1 preimages, is exactly the boundary $\partial \mathscr{B}(\infty)$, i.e. all the points inside it generate bounded trajectories. This is due to the fact that there are no other preimages of $\omega_{1}$ and $\omega_{2}$ because $\omega_{1}^{-1}$ and $\omega_{2}^{-1}$ belong to a region of the plane whose points have no preimages. This fact can be clearly seen by the study of the critical curves of the noninvertible map (17) (see e.g. $[11,18])$.

\subsection{Critical curves}

The map (17) is a noninvertible endomorphism because if, in (17), the point $\left(x_{1}, x_{2}\right)$ is computed in terms of a given $\left(x_{1}^{\prime}, x_{2}^{\prime}\right)$ a fourth degree algebraic system is obtained, that can have four, two or no 


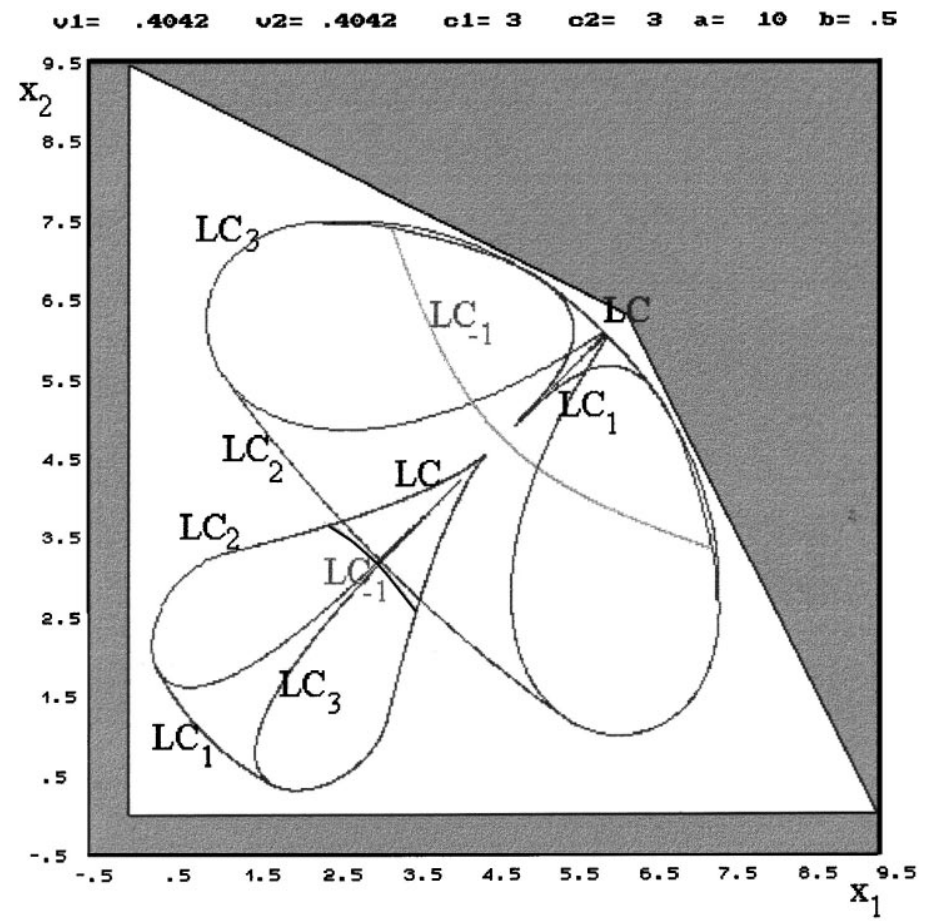

Fig. 3. The absorbing area $S$ bounded by segments of critical curves of rank $1,2,3$, 4 , denoted by $L C, L C_{1}, L C_{2}$, and $L C_{3}$, respectively (each of these segments of critical curves is formed by two branches, being images of increasing rank of the two branches of $L C_{-1}$ shown in the figure).

solutions. As the point $\left(x_{1}{ }^{\prime}, x_{2}{ }^{\prime}\right)$ varies in the plane $\mathbb{R}^{2}$ the number of solutions, i.e. the number of its real rank-one preimages, can change: pairs of real preimages appear or disappear as the point $\left(x_{1}{ }^{\prime}, x_{2}{ }^{\prime}\right)$ crosses the curves separating regions characterized by different number of preimages. Such curves are characterized by the presence of two coincident (merging) preimages. This leads to the definition of the critical curves, one of the distinguishing features of noninvertible maps. The critical curve of rank-1, denoted by $L C$, is defined as the locus of points having two, or more, coincident rank-1 preimages, located on a set called $L C_{-1} . L C$ is the two-dimensional generalization of the notion of critical value (when it is a local minimum or maximum value) of a one-dimensional map, $L C_{-1}$ is the generalization of the notion of critical point (when it is a local extremum point). Arcs of $L C$ separate the regions of the plane characterized by a different number of real preimages (see $[11,18,1]$ ). We also recall that the critical sets of rank $k$, are the images of rank $k$ of $L C_{-1}$ denoted by $L C_{k-1}=T_{s}^{k}\left(L C_{-1}\right)=$ $T_{s}^{k-1}(L C), L C_{0}$ being $L C$.

Being $L C_{-1}$ the locus of coincident rank-1 preimages of the points of $L C$, in any neighborhood of a point of $L C_{-1}$ there are at least two distinct points mapped by $T_{s}$ in the same point near $L C$. Hence, the map $T_{s}$ is not locally invertible in the points of $L C_{-1}$ and, since the map (17) is a continuously differentiable map, this implies that $L C_{-1}$ belongs to the set of points where the Jacobian determinant of $T_{s}$ vanishes, i.e.

$$
L C_{-1}=\left\{\mathbf{x} \in \mathbb{R}^{2} \mid \operatorname{det} D T_{s}=0\right\}
$$


and $L C$ is the rank-1 image of $L C_{-1}$ under $T$, i.e. $L C=T_{S}\left(L C_{-1}\right)$ (see $[11,18]$ ). From (23), the condition $\operatorname{det} D T_{s}=0$ becomes

$$
x_{1}^{2}+x_{2}^{2}+4 x_{1} x_{2}-\alpha x_{1}-\alpha x_{2}+\beta=0
$$

with

$$
\alpha=\frac{5+5 b v^{2}(a-c)}{4 b^{2} v^{2}} \text { and } \beta=\frac{\left(1+b v^{2}(a-c)\right)^{2}}{4 b^{2} v^{2}}
$$

This is the equation of an hyperbola in the plane $\left(x_{1}, x_{2}\right)$, hence $L C_{-1}$ is formed by two branches, denoted by $L C_{-1}^{(a)}$ and $L C_{-1}^{(b)}$ in Fig. 2. This implies that also $L C$ is the union of two branches, denoted by $L C^{(a)}=T_{s}\left(L C_{-1}^{(a)}\right)$ and $L C^{(b)}=T_{s}\left(L C_{-1}^{(b)}\right)$. Each branch of the critical curve $L C$ separates the phase plane of $T$ into regions whose points have the same number of distinct rank-1 preimages. In the case of the map (10) $L C^{(b)}$ separates the region $Z_{0}$, whose points have no preimages, from the region $Z_{2}$, whose points have two distinct rank-1 preimages, and $L C^{(a)}$ separates the region $Z_{2}$ from $Z_{4}$, whose points have four distinct preimages. Notice that $L C_{-1}^{(b)}$ intersects the diagonal $\Delta$ in the critical point of the restriction $f$, i.e. $L C_{-1}^{(b)} \cap \Delta=((1+v(a-c)) / 6 b v,(1+v(a-c)) / 6 b v)$ and consequently $L C^{(b)}$ intersects $\Delta$ in the point where the restriction $f$ attains its maximum value.

As far as the segments $\omega_{1}^{-1}$ and $\omega_{2}^{-1}$ entirely belong to the region $Z_{0}$, as in the situation shown in Fig. 2, no other portions of $\partial \mathscr{B}(\infty)$ are obtained by higher rank preimages of $\omega_{1}$ and $\omega_{2}$. This implies that according to (30), $\partial \mathscr{B}(\infty)$ has the simple structure of the quadrilateral shown in the Fig. 2, Fig. 3, Fig. 4(a). But the situation radically changes if some portions of $\omega_{1}^{-1}$ or $\omega_{2}^{-1}$ enter $Z_{2}$ as some parameter is changed, as we shall see in Section 4.

\section{Intermittency and attracting area}

For $v(a-c) \in(0,2)$, the fixed point $E_{*}$ is asymptotically stable and its basin of attraction is the region $\mathscr{D}$ inside the quadrilateral $O O_{-1}^{(1)} O_{-1}^{(3)} O_{-1}^{(2)}$. For $v(a-c)>2 E_{*}$ is a saddle, with local unstable set along $\Delta$ and local stable set transverse to it (tangent to the orthogonal direction). As recalled in Section 2, if $\mathrm{v}(\mathrm{a}-\mathrm{c}) \in[2,3]$ the attractor $\mathscr{A}$ of $f=\left.T_{s}\right|_{\Delta}$, which may be a cycle, a cycle of chaotic intervals or a weak attractor given by a Cantor set, belongs to the attracting set $I_{\Delta}=C_{1} C \subset \Delta . I_{\Delta}$ is also a one-dimensional trapping set for the two-dimensional map $T_{s}$ if all the cycles inside it are transversally attracting. In fact, in this case all the trajectories starting inside a two-dimensional neighborhood $U$ of $I_{\Delta}$ synchronize and their $\omega$-limit set is the attractor $\mathscr{A} \subseteq I_{\Delta}$. Moreover, if no other bounded attracting sets exist outside $\Delta$ then all the trajectories starting in the basin $\mathscr{D}$ of bounded trajectories, defined as the complementary set of the closure of $\mathscr{B}(\infty)$

$$
\mathscr{D}=C(\overline{B(\infty)})
$$

synchronize and converge to $\mathscr{A}$. In the simple cases shown in Figs. 2, 3 and 4 a the set $\mathscr{D}$ is the region inside the quadrilateral $O O_{-1}^{(1)} O_{-1}^{(3)} O_{-1}^{(2)}$.

As $v$ increases, a local bifurcation value $v_{b}$ is reached at which a $k$-cycle located on $\Delta$ becomes transversally unstable. For example, with the parameters $a, b, c$ used in Fig. 1, Fig. 2 and Fig. 3 $v_{b} \simeq 0.37563$ and $k=2$, i.e. as $v$ increases beyond $v_{b}$ a 2-cycle located on $\Delta$ becomes transversally 

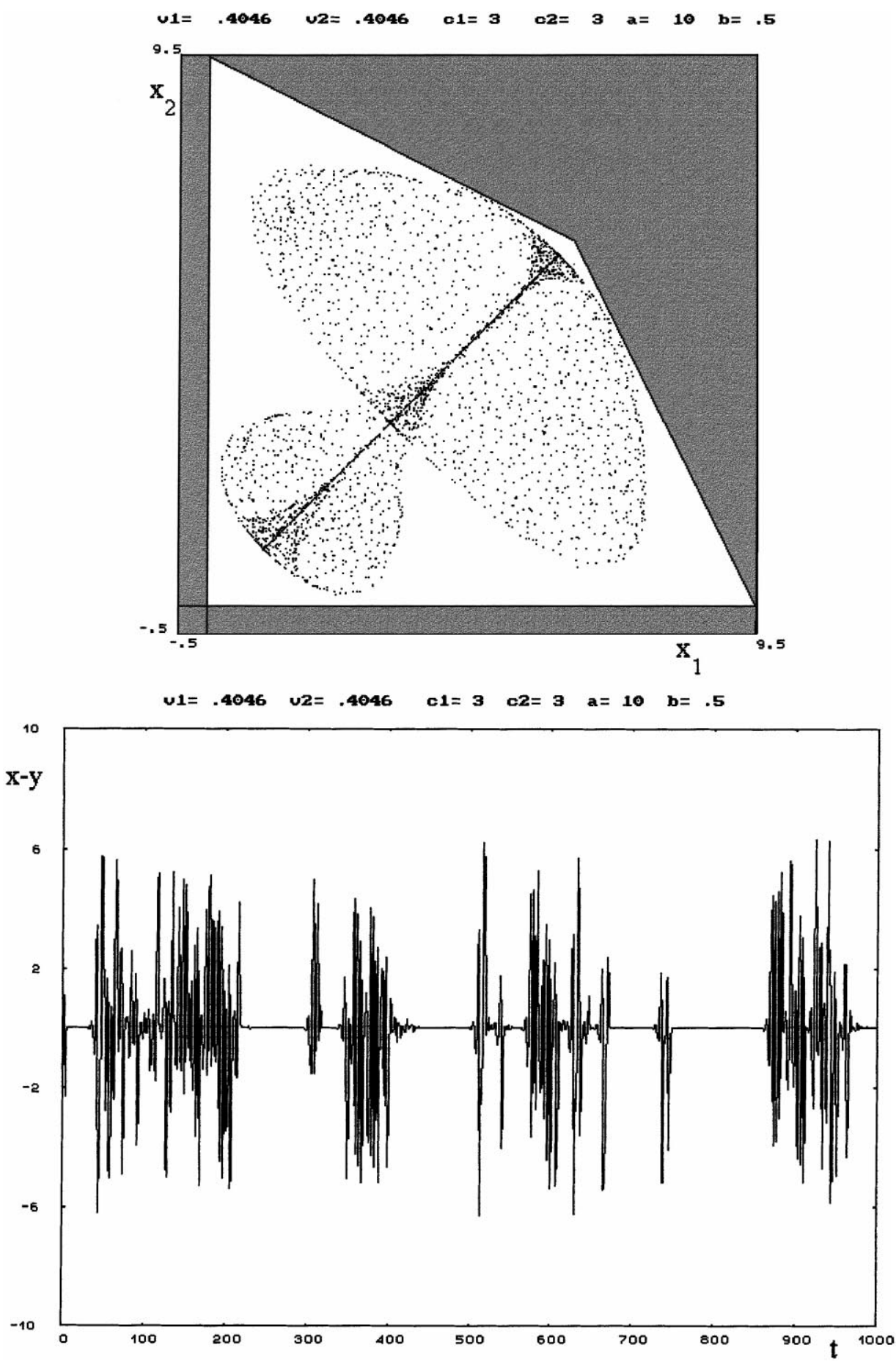

Fig. 4. For parameters' values $c=3, a=10, b=0.5$ (as in the previous figures) and $v=0.4046$ a typical trajectory starting in the white region is shown both in the phase space (a) and versus time (b). From the graph versus time the intermittent behavior can be clearly seen, and the figure in the phase space shows that the intermittency is bounded inside the absorbing area $S$. 
unstable with $\lambda_{\perp}^{(2)}<-1$. For $v<v_{b}$, just before the bifurcation, this 2-cycle is a saddle, repelling along $\Delta$ and transversally attracting, with $-1<\lambda_{\perp}^{(2)}<0$. At $v=v_{b}$, it becomes a repelling node via a flip bifurcation, at which a saddle cycle of period 4 is created out of $\Delta$, with periodic points located symmetrically with respect to the diagonal. For $v>v_{b}$ the invariant interval $I$ is no longer an asymptotically stable attracting set for the map $T_{s}$. In fact, if we consider a sufficiently small neighborhood $U$ of $I$ we have that the local unstable set of the transversally unstable $k$-cycle intersects $\partial U$ and a set of points of positive Lebesgue-measure exists in $U$ whose trajectories exit $U$ in a finite number of iterations. This implies that no neighborhood $V \subset U$ exists such that the definition of Lyapunov stability holds. In this case it is possible to prove that around the local transverse unstable set of the 2-cycle open sets of points exist, called 'tongues' in [13] or 'wedges'in [2], such that the trajectories starting from points of these 'tongues' exit $U$ after a finite number of iterations. Similar 'tongues' also exist in correspondence of the infinite preimages, along $\Delta$, of the points of the 2-cycle. Thus, for $v>v_{b}$, two question arise:

- what is the fate of the trajectories that, even starting very close to $I_{\Delta}$, exit the neighborhood $U$ ?;

- what is the asymptotic behavior of the generic trajectory starting inside the basin of bounded trajectories $\mathscr{D}$ ?.

Some answers to these questions can be obtained from a study of the global properties of the map $T_{s}$, and for a noninvertible map these properties can be characterized by the use of critical curves. In fact, even if for $v>v_{b}$ the invariant interval $I_{\Delta}$ ceases to be a topological attracting set of $T_{s}$, a two dimensional attracting set $S$ is created around $I$, whose boundary $\partial S$ is formed by a finite number of arcs of critical curves $L C_{k}, k=1, \ldots, m$. This is an invariant set of $T_{s}$, i.e. $T_{s}(S) \equiv S$, such that every trajectory starting inside $\mathscr{D}$ enters $S$ after a finite number of iterations, and its boundary can be obtained following the procedure described in [18], ch. 4: if $\gamma=S \cap L C_{-1}$ then, for a suitable integer $m$

$$
\partial S \subseteq \bigcup_{k=1}^{m} T^{k}(\gamma)
$$

An example is shown in Fig. 3, where $m=4$. We remark that $S$ includes the invariant interval $I_{\Delta}$, being $S \cap \Delta=I_{\Delta}$, and all the trajectories starting inside a neighborhood of $I$ inside $S$ cannot go out of $S$. Loosely speaking $\partial S$ behaves as a bounded vessel for the trajectories starting from the 'tongues' located around the local unstable sets of the transversally repelling cycles.

For $v>v_{b}$ other $k$-cycles of $f$, with $k \geq 2$, belonging to $\Delta$, become transversally unstable. This can occur either through a flip bifurcation if $\lambda_{\perp}^{(k)}<0$ (as in the case of the 2-cycle considered above) or a fold bifurcation if $\lambda_{\perp}^{(k)}>0$.

If $T_{s}$ has no attractors outside of $\Delta$ then the generic trajectory starting inside $\mathscr{D}$ synchronizes after a few bursts away from $\Delta$ and eventually is attracted by $\mathscr{A} \subseteq I_{\Delta}$, whereas if $T_{s}$ has attractors outside of $\Delta$ then trajectories starting arbitrarily close to $I_{\Delta}$ may exist that converge to them. However, such attractors must necessarily be inside $S$.

Interesting phenomena, well studied in the recent literature, occur when the parameters values are such that $\mathscr{A}$ is a chaotic interval or a cycle of chaotic intervals, i.e. chaotic synchronization takes place. For example, at $v=\overline{v_{0}}=\left(\overline{\mu_{0}}-1\right) /(a-c)$, with $\overline{\mu_{0}}=3.678573510428 \ldots{ }^{2}, f$ is chaotic on $\mathscr{A}=I_{\Delta}$ and

\footnotetext{
${ }^{2}$ For the logistic map (20) $\overline{\mu_{0}}$ is the parameters' value of the first homoclinic bifurcation of the fixed point, at which two invariant intervals merge to give a unique chaotic interval, as recalled in Section 2.2.
} 
has absolutely continuous invariant measure on $I_{\Delta}$. The repelling 2-cycle is contained in $\mathscr{A}$, and the infinitely many preimages of such a cycle are dense in $\mathscr{A}$. Moreover, the fact that $L_{\perp}<0$ at $v=\overline{v_{0}}$ (see Fig. 1) ensures that $\mathscr{A}$ is an attractor in the weak Milnor sense. This means that $\mathscr{A}$ attracts a set of points of positive measure in any neighborhood $U_{\delta}(\mathscr{A})$, where

$$
U_{\delta}(\mathscr{A})=\left\{\left(x_{1}, x_{2}\right) \mid\left\|\left(x_{1}-x, x_{2}-x\right)\right\|<\delta \forall x \in \mathscr{A}\right\}
$$

Furthermore, in this case $L_{\lambda}<0$ implies (see [2]) that $\mathscr{A}$ is an essential attractor for $T_{s}$, i.e.

$$
\lim _{\delta \rightarrow 0} \frac{\mu\left(\mathscr{B}(A) \cap U_{\delta}(A)\right)}{\mu\left(U_{\delta}(A)\right)}=1
$$

where $\mu$ is a Lebesgue measure on $\mathbb{R}^{2}$. In simpler words, most initial conditions close to $\mathscr{A}$ are attracted to $\mathscr{A}$, but in any neighborhood of $A$ there exists a dense set that is locally repelled in a direction transverse to $\Delta$. In this situation, according to the fate of the locally repelled trajectories, determined by the global dynamics of the system, two different cases can be distinguished, assuming that the absorbing area $S$ exists and $L_{\perp}<0$ :

- case I. The locally repelled trajectories eventually return to $A$ (i.e. synchronize) after a transient phase in which they make several excursions (bursts) away of $\Delta$ (but necessarily inside $S$ ). If $L_{\perp}$ is negative but sufficiently close to zero then this transient may be very long, and the phenomenon called on-off intermittency is obtained. An upper bound to the amplitude of such bursts is determined by $\partial S$ (an intermittent trajectory is shown in Fig. 4, in phase space (4a) and versus time (4b)).

- case II. The locally repelled trajectories belong to the basin of some other attractor outside of $\Delta$. In this case, the basin of $A$ is riddled with the basin of the other attractor (according to [2], $\mathscr{B}(A)$ is called 'riddled basin' if it has positive measure but every point of $\mathscr{B}(A)$ is such that any neighborhood of it contains points whose trajectory converge to another attractor).

We remark that as far as the absorbing area $S$ exists, the dynamics are in any case confined inside $S$. This is the role of the global properties of the map $T_{s}$. Loosely speaking we can say that the local unstable manifolds of the transversally repelling cycles of $\Delta$ are folded back by the folding action of the critical curves.

We also remark that Case II has not been observed by the numerical explorations performed with our map (17), but we cannot exclude it, and examples of its occurrence are given in the literature $([10,15])$.

The importance of the invariant area $S$ is even more evident if a small parameters' mismatch

$$
v_{2}=v_{1}+\varepsilon \text { and } / \text { or } c_{2}=c_{1}+\delta
$$

where the constants $\varepsilon$ and $\delta$ are small in comparison with the values of the respective parameters, is introduced. If $\left(v_{1}, c_{1}\right) \neq\left(v_{2}, c_{2}\right)$ then the diagonal $\Delta$ is no longer invariant because the symmetry property of the map is lost. The destruction of the invariant set $\mathscr{A} \subset \Delta$ implies that every trajectory starting inside the basin of $S$ fills up the two-dimensional attracting set $S$. For example, in the Fig. 5(a) and (b) two trajectories are represented, both starting from the basin of $S$, one obtained in the symmetric case (9) of identical players, with $v_{1}=v_{2}=0.4035$ and $c_{1}=c_{2}=3$, and the other obtained after the introduction of a very small difference between the marginal costs, namely $c_{1}=3$ and $c_{2}=3.00001$. 

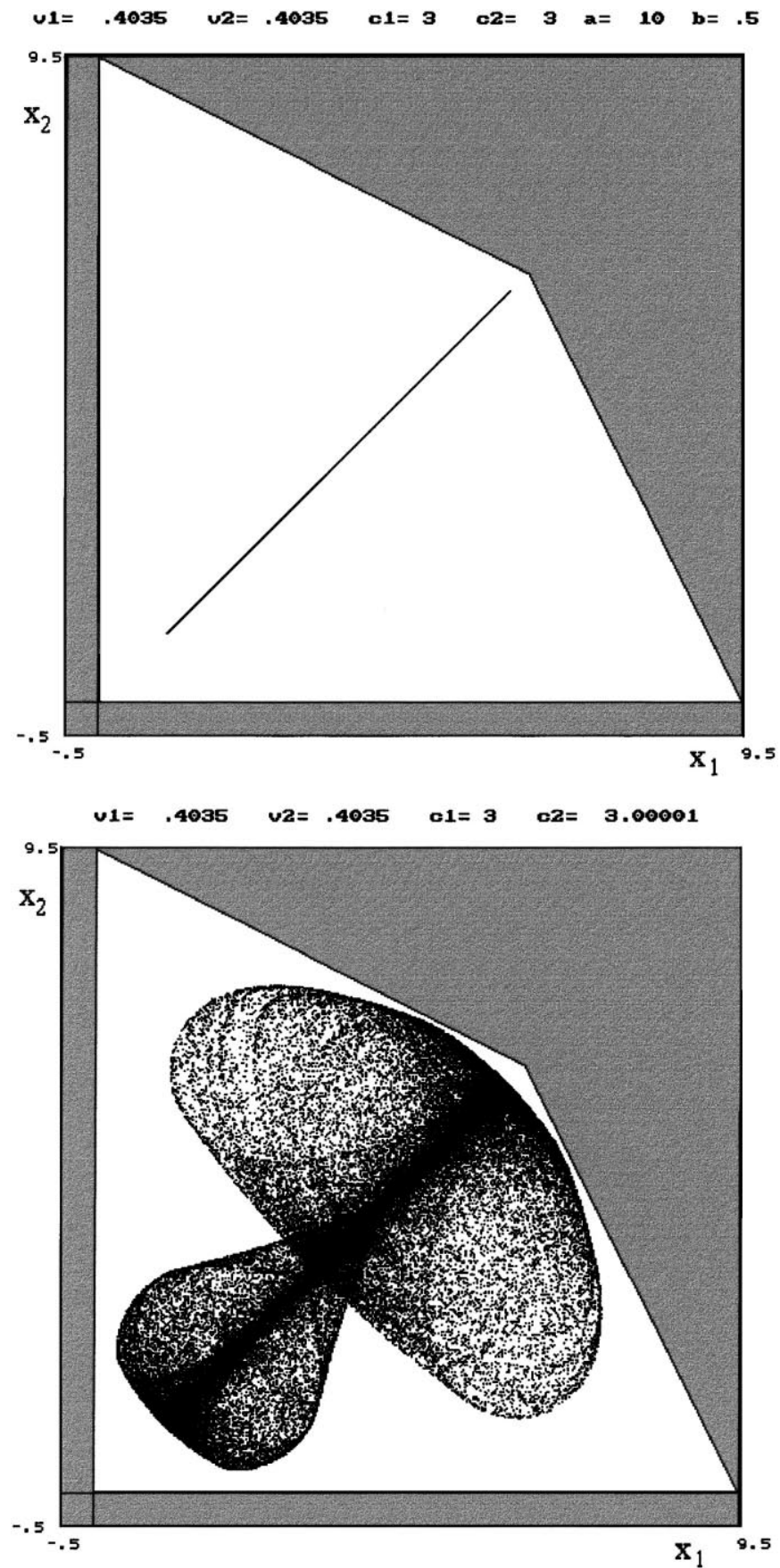

Fig. 5. Effect of a symmetry-breaking caused by a small parameters' mismatch. In Fig. (a), obtained with identical parameters characterizing the two players, $v_{1}=v_{2}=0.404$ and $c_{1}=c_{2}=3$, the $\omega$-limit set of a generic trajectory starting from the white region is the one-dimensional Milnor attractor along the diagonal on which synchronized trajectories take place. In Fig. (b), obtained with $v_{1}=v_{2}=0.404, c_{1}=3$ and $c_{2}=3.00001$, the $\omega$-limit set of a generic trajectory starting from the white region covers the whole two-dimensional attractor $S$, inside which the trajectory shows an intermittent behavior. 
For the set of parameters used in Fig. 5(a) the Milnor attractor $\mathscr{A}$ on the diagonal is not a topological attractor since $v>v_{b}$, but it is an essential attractor because $L_{\perp}=-0.115$. Despite of the very small difference between the parameters of the two producers, the asymptotic behavior is very different, since the trajectory of Fig. 5(a) synchronizes after a transient of 500 iterations (not shown in the figure), whereas the trajectory of Fig. 5(b) continue to move erratically, with intermittent bursts away from the diagonal, but always inside the absorbing area $S$. Indeed, it can be noticed that inside the large chaotic area the points near the diagonal $\Delta$ are more frequently visited than those far from it. Such transition from the asymptotic synchronization to the continual sequence of intermittent bursts, due to a parameters' mismatch (no matter how small) has been observed by many authors (see [21,24,25]) and is called hard bubbling transition in [24]. However, in these papers no results are given about bounds to the amplitude of the bursts.

The study of the effects of small asymmetries is of noticeable interest in the modeling of real economic systems since small heterogeneities between the economic agents are always present (see [6]).

If a chaotic attractor $\mathscr{A}$ exists on $\Delta$ with $L_{\perp}>0$ then almost every initial condition is locally repelled away from $\mathscr{A}$, even if there exists a subset of infinitely many points of $\mathscr{A}$ that are locally attracting in the transverse direction (in our example this is certainly true for the fixed point $E_{*}$, whose transverse eigenvalue is given in (26), together with all its preimages belonging to $\Delta$ ). In this case, the generic trajectory starting inside the set $\mathscr{D}$, even very close to $\Delta$, fills up the invariant area $S$, showing an intermittent behavior, and $S$ is the only attractor that can be numerically seen.

\section{Global bifurcations}

In this section, we examine some global bifurcations, typical of noninvertible maps, related to contacts of the critical curves with the boundary of $\mathscr{B}(\infty)$. Such contact bifurcations, occurring far from the invariant submanifold $\Delta$ where synchronized dynamics take place, can have an influence on the destiny of the locally repelled trajectories. This puts in evidence the role of the global dynamical properties in the questions addressed in Section 3.

\subsection{Bifurcation changing $\mathscr{B}(\infty)$ from connected to non connected}

As the parameter $v$ is increased the branch of rank-1 critical curve $L C^{(\mathrm{b})}$, which separates $Z_{0}$ from $Z_{2}$, moves upwards and a value of $v$ exists, say $v_{H}$, at which it has a contact (or tangency) with $\omega_{1}^{-1}$ and $\omega_{2}^{-1}$ (the contacts with the two sides $\omega_{1}^{-1}$ and $\omega_{2}^{-1}$ of $\partial \mathscr{B}(\infty)$ occur simultaneously because of the symmetry, with respect to the diagonal $\Delta$, both of $L C$ and $\partial \mathscr{B}(\infty)$ ). For example, with the same values of the parameters $a, b, c$ as those used to obtained the Figs. 1-4, such contact occurs at $v=v_{H} \simeq 0.4047476$. This contact marks the occurrence of a global bifurcation that causes the transformation of $\mathscr{B}(\infty)$ from connected to non connected, according to the following proposition.

Proposition 2. At $v=v_{H}$ the basin $\mathscr{B}(\infty)$ is transformed from connected to non connected, and for $v_{H}<v<(3 / a-c) \mathscr{B}(\infty)$ is given by the region out of the quadrilateral $O O_{-1}^{(1)} O_{-1}^{(3)} O_{-1}^{(2)}$ and an infinite sequence of non connected regions (or holes) inside the quadrilateral. 


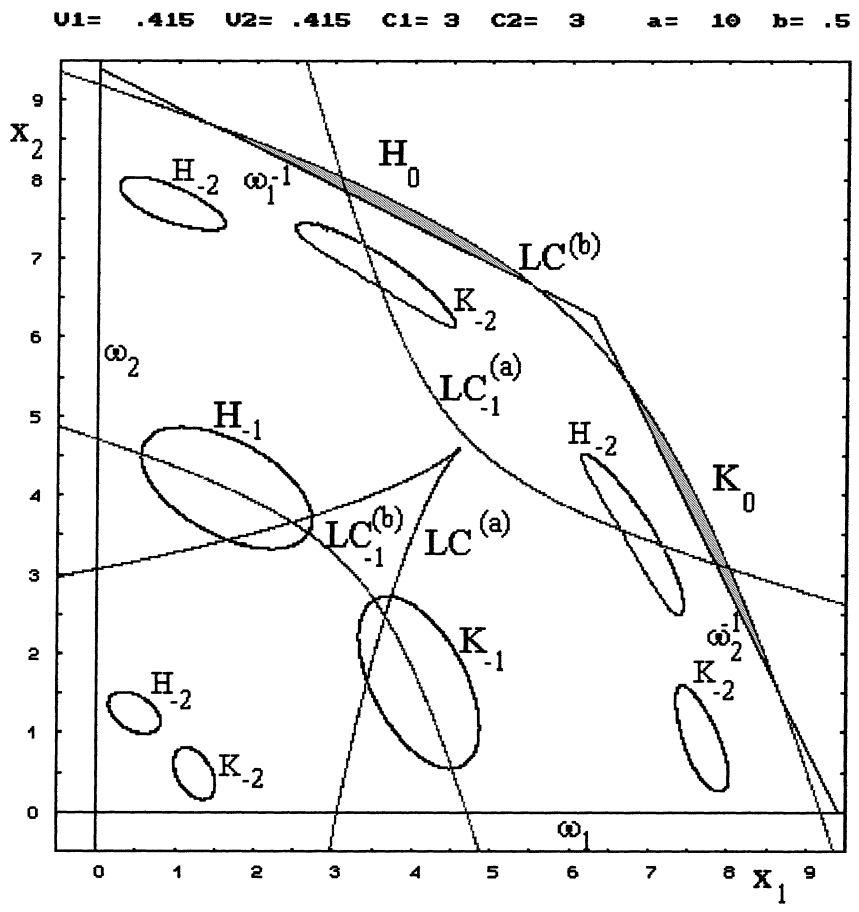

Fig. 6. Some preimages of the portions of $\omega_{1}$ and $\omega_{2}$ of the invariant axes. The regions $\mathrm{H}_{0}$ and $\mathrm{K}_{0}$ denote the portions of the immediate basin of infinity included inside the region $Z_{2}$. Their rank-1 preimages are the main holes, denoted by $\mathrm{H}_{-1}$ and $\mathrm{K}_{-1}$, bounded by preimages of rank-1 of $\omega_{1}^{-1}$ and $\omega_{2}^{-1}$, respectively. Preimages of rank-2 of $\omega_{1}^{-1}$ and $\omega_{2}^{-1}$ are also shown in the figure. Other infinitely many holes, bounded by preimages of higher rank, exist, but are not represented in the figure.

This is a well known bifurcation (see e.g. [17,18] ch. 5, [1] ch.5). For $v>v_{H}$, just after the contact bifurcation, two portions of $\mathscr{B}(\infty)$, say $\mathrm{H}_{0}$ and $\mathrm{K}_{0}$ (bounded by $L C^{(\mathrm{b})}$ and segments of $\omega_{1}^{-1}$ and $\omega_{2}^{-1}$, respectively) enter inside $Z_{2}$ (see Fig. 6)

Hence for $v>v_{H}$ the expression (30) giving the boundary $\partial \mathscr{B}(\infty)$, does not contain only rank-1 preimages of $\omega_{1}$ and $\omega_{2}$, but also preimages of higher rank. In fact, the points belonging to $\mathrm{H}_{0}$, as well as those belonging to $\mathrm{K}_{0}$, have two distinct preimages, located at opposite sides with respect to the line $L C_{-1}^{(b)}$, with the exception of the points of the curve $L C^{(b)}$ inside $\mathscr{B}(\infty)$ whose preimages, according with the definition of $L C$, merge on $L C_{-1}^{(b)}$. Since $\mathrm{H}_{0}$ and $\mathrm{K}_{0}$ are part of $\mathscr{B}(\infty)$ also their preimages belong to $\mathscr{B}(\infty)$ and form holes (or lakes, following [17]) which, for $v_{H}<v<(3 / a-c)$, are non connected portions of $\mathscr{B}(\infty)$ inside the quadrilateral $O O_{-1}^{(1)} O_{-1}^{(3)} O_{-1}^{(2)}$. In fact, only at $v=v_{f}=3 / a-c \mathrm{H}_{0}$ and $\mathrm{K}_{0}$, as well as their rank-1 preimages $\mathrm{H}_{-1}$ and $\mathrm{K}_{-1}$, have a contact with the coordinate axes (as will be explained in Section 4.2). The two main holes $\mathrm{H}_{-1}$ and $\mathrm{K}_{-1}$ lie entirely inside the regions $Z_{2}$ and $Z_{4}$, hence each of them has further preimages, which are smaller holes of $\mathscr{B}(\infty)$ bounded by preimages of rank 2 of $\omega_{1}$ and $\omega_{2}$. Even these lie inside $Z_{2}$ and $Z_{4}$, so each of them has further preimages, and so on. These preimages are infinitely many because from the property $T_{s}\left(O O_{-1}^{(1)} O_{-1}^{(3)} O_{-1}^{(2)}\right) \supseteq O O_{-1}^{(1)} O_{-1}^{(3)} O_{-1}^{(2)}$

\footnotetext{
${ }^{3}$ The value of the parameter $v$ is used in Fig. 6 is much greater than the bifurcation value $v_{H}$ in order to make the figure more readable, since for values of $v$ just after the bifurcration the holes are very small.
} 


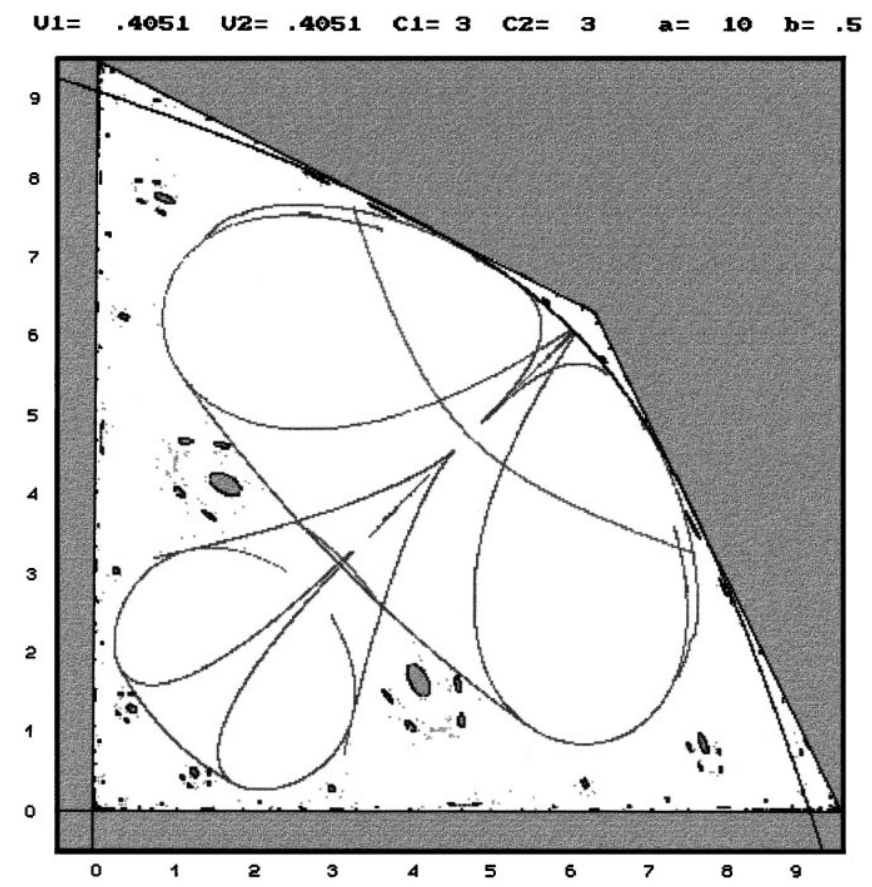

Fig. 7. This figure is obtained just after the basin bifurcation due to the contact between $L C^{(b)}$ and the segments $\omega_{1}^{-1}$ and $\omega_{2}^{-1}$. The infinitely many holes created at the contact bifurcation accumulate on the repelling cycles located on the invariant coordinate axes and on other repelling cycles existing out of the absorbing area $S$.

follows that at least an infinite sequence of preimages of the main holes $\mathrm{H}_{-1}$ and $\mathrm{K}_{-1}$ must exist inside the quadrilateral (see e.g. [18], ch. 4).

Thus, after the bifurcation value $v_{H}$ infinitely many disjoint portions of $\mathscr{B}(\infty)$ appear inside the quadrilateral $O O_{-1}^{(1)} O_{-1}^{(3)} O_{-1}^{(2)}$, and the boundary $\partial \mathscr{B}(\infty)$ is given by the quadrilateral $O O_{-1}^{(1)} O_{-1}^{(3)} O_{-1}^{(2)}$ and the infinite sequence of boundaries of the holes, given by the infinitely many preimages of any rank of the portions of $\omega_{1}^{-1}$ and $\omega_{2}^{-1}$ included in $\mathrm{H}_{0}$ and $\mathrm{K}_{0}$. It can be observed that holes inside the region $Z_{4}$ also exist. These have four distinct preimages, pairwise located at opposite sides with respect to $L C_{-1}^{(a)}$ and $L C_{-1}^{(b)}$. Some other holes, like the main holes $\mathrm{H}_{-1}$ and $\mathrm{K}_{-1}$ of Fig. 6, belong to both the regions $Z_{2}$ and $Z_{4}$ and include a segment of $L C^{(a)}$ inside, hence they have preimages merging along $L C_{-1}^{(a)}$.

The invariant area $S$, bounded by portions of critical curves according to (36), is not destroyed by this contact bifurcation. This is due to the fact that the portions of $L C$ that are involved in the contact with $\partial \mathscr{B}(\infty)$ do not belong to the boundary of the absorbing area. The infinitely many holes, being given by sequences of preimages, accumulate on repelling sets of the map $T_{s}$. For example, in the situation shown in Fig. 7, they accumulate on the repelling cycles located on the invariant coordinate axes and on other repelling cycles existing inside $\mathscr{D}$, out of the absorbing area $S$. We remark that the holes do not enter inside the area $S$ because the points of $L C$ in which the contact with $\partial \mathscr{B}(\infty)$ occurs do not belong to the portions of $L C$ bounding $S$, given, according to (36) by $T(\gamma)$, where $\gamma=L C_{-1} \cap S$.

As $v$ is further increased $L C$ continues to move upwards, the portions $\mathrm{H}_{0}$ and $\mathrm{K}_{0}$ grow up and consequently the holes become larger. This fact causes a sort of loss of predictability, since a greater 
uncertainty is obtained with respect to the asymptotic evolution of games starting from an initial strategy taken in regions out of $S$ with many holes.

\subsection{Contact bifurcation that destroys $S$}

The invariant area $S$ is destroyed when some holes have a contact with its boundary $\partial S$. Such a contact must necessarily occur as the parameter $v$, and/or the difference $(a-c)$ are increased. In fact for $v(a-c)=3$ the main holes $\mathrm{H}_{-1}$ and $\mathrm{K}_{-1}$, as well as their preimages of any rank, are tangent to the diagonal $\Delta$, as we shall see in next subsection. However, the destruction of $S$ occurs before this, when a portion of $\partial \mathscr{B}(\infty)$ has a contact with $\partial S$. When this happens also the preimages of such portion of $\partial \mathscr{B}(\infty)$ have contacts with $\partial S$ hence, after the bifurcation, infinitely many holes enter $S$. Of course, this implies that $S$ is no longer invariant after this bifurcation.

Let us denote by $v_{c}$ the value of the parameter $v$ at which $\partial S$ has the first contact with $\partial \mathscr{B}(\infty)$. For the set of parameters $a, b, c$ given above we have $v_{c} \simeq 0.4051$ and the bifurcation is due to a contact between the portion of $L C_{3}$ bounding the upper part of $S$ and the sides $\omega_{1}^{-1}$ and $\omega_{2}^{-1}$ of $\partial \mathscr{B}(\infty)$.

For $v_{H}<v<v_{c}$, infinitely many holes exist inside the quadrilateral $O O_{-1}^{(1)} O_{-1}^{(3)} O_{-1}^{(2)}$, but a large region exists, occupied by the invariant area $S$, without holes inside. For $v>v_{c}$, the region inside the quadrilateral $O O_{-1}^{(1)} O_{-1}^{(3)} O_{-1}^{(2)}$ becomes almost filled up with holes of $\mathscr{B}(\infty)$, and the set $\mathscr{D}$, defined in (35), whose points generate bounded trajectories, assumes a very complex structure, called 'fat fractal' in [20], characterized by the presence of infinitely many holes distributed all over it.

In our example, this contact bifurcation marks the passage from case (I) to case (II) described in Section 3, i.e. between the phenomenon of intermittency and that of riddled (apparently riddled on the basis of numerical computations) basins.

If we consider a value $\bar{v} \in\left(v_{c}, 3 / a-c\right)$ at which a chaotic attractor $\mathscr{A}$ of $\left.T_{s}\right|_{\Delta}$, with absolutely continuous invariant measure, exists inside $I_{\Delta}$, and we know that infinitely many of such values can be found, then at $v=\bar{v}$ the basin $\mathscr{B}(\mathscr{A})$ may be riddled with $\mathscr{B}(\infty)$ provided that $L_{\perp}<0$ (see [2]). In Fig. 1 it can be noticed that in the range $\left(v_{c}, 3 / a-c\right.$ ) intervals can be identified where $L_{\perp}<0$ (see the enlargement in Fig. 8(a)). With the value $v=0.411$, where $L_{\perp}<0, \mathscr{B}(\mathscr{A})$ has been numerically represented in Fig. 8(b). For this set of parameters the attractor $\mathscr{A}$ is a one-piece chaotic attractor inside $I_{\Delta}$. For each initial condition taken over a grid on the portion of phase plane represented in the figure a trajectory of the map $T_{s}$ has been generated and a grey dot is painted if the trajectory reaches the immediate basin of infinity (i.e. the region outside the quadrilateral $O O_{-1}^{(1)} O_{-1}^{(3)} O_{-1}^{(2)}$ ), whereas a white point is painted if after $10^{6}$ iterations the inequality $\left|x_{1}-x_{2}\right|<10^{-7}$ is satisfied. At a first look to Fig. 8(b) the structure of $\mathscr{B}(\mathscr{A})$, represented by the distribution of white points, looks rather complicated, similar to that of a riddled basin. The appearance of the Fig. 8(b) remains the same even if a longer transient is discarded before the test for synchronization is performed, and similar structures are seen if enlargements are considered. The figures obtained suggests that $\mathscr{B}(\mathscr{A})$ is given by the complementary of the closure of all the infinitely many holes of $\mathscr{B}(\infty)$ inside the quadrilateral $O O_{-1}^{(1)} O_{-1}^{(3)} O_{-1}^{(2)}$.

Instead, if values of $v \in\left(v_{c}, 3 / a-c\right)$ are taken such that $L_{\perp}>0$ then almost all the trajectory are repelled from $\Delta$ and then diverge, i.e. completely grey figures are obtained following the numerical procedure used to obtain Fig. 8(b). In these cases, if a chaotic attractor $\mathscr{A}$ of $\left.T_{s}\right|_{\Delta}$ exists, it is a chaotic saddle for the two-dimensional map $\left.T_{s}\right|_{\Delta}$, i.e. $\mu(\mathscr{B}(\mathscr{A}))=0$ (see [4]). We notice, however, that a subset of zero measure of points that are attracted to $\Delta$ exist since there are cycles (that may also be infinitely 
(a)

$c=3 \quad a=10 \quad b=.5$

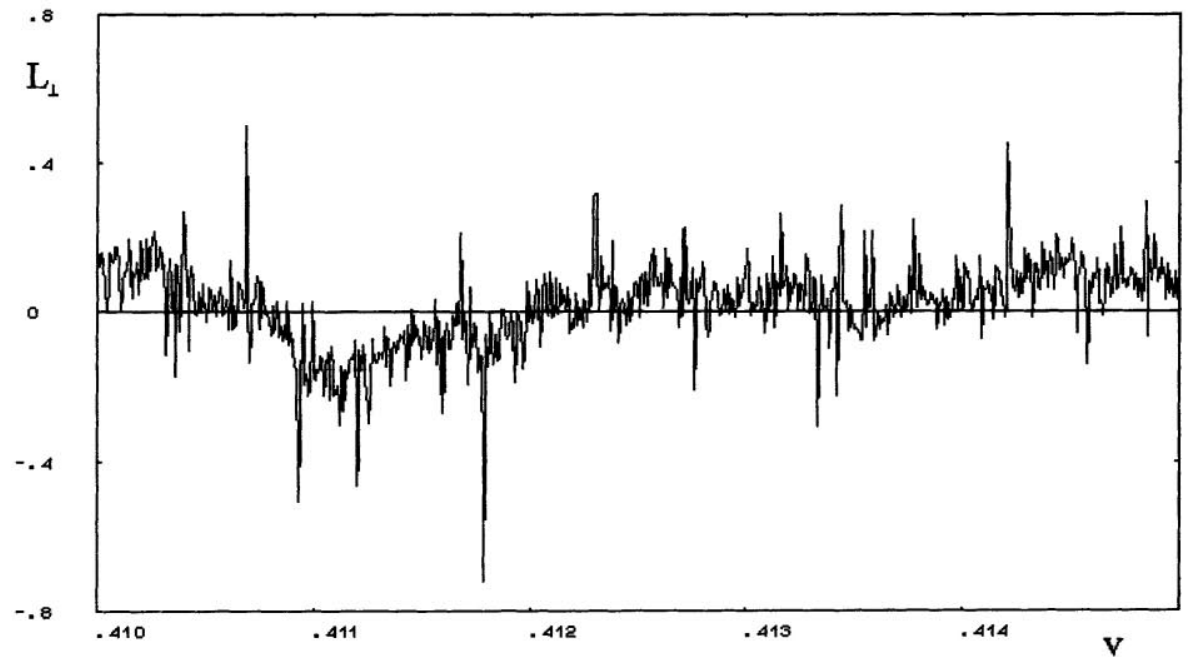

(b)

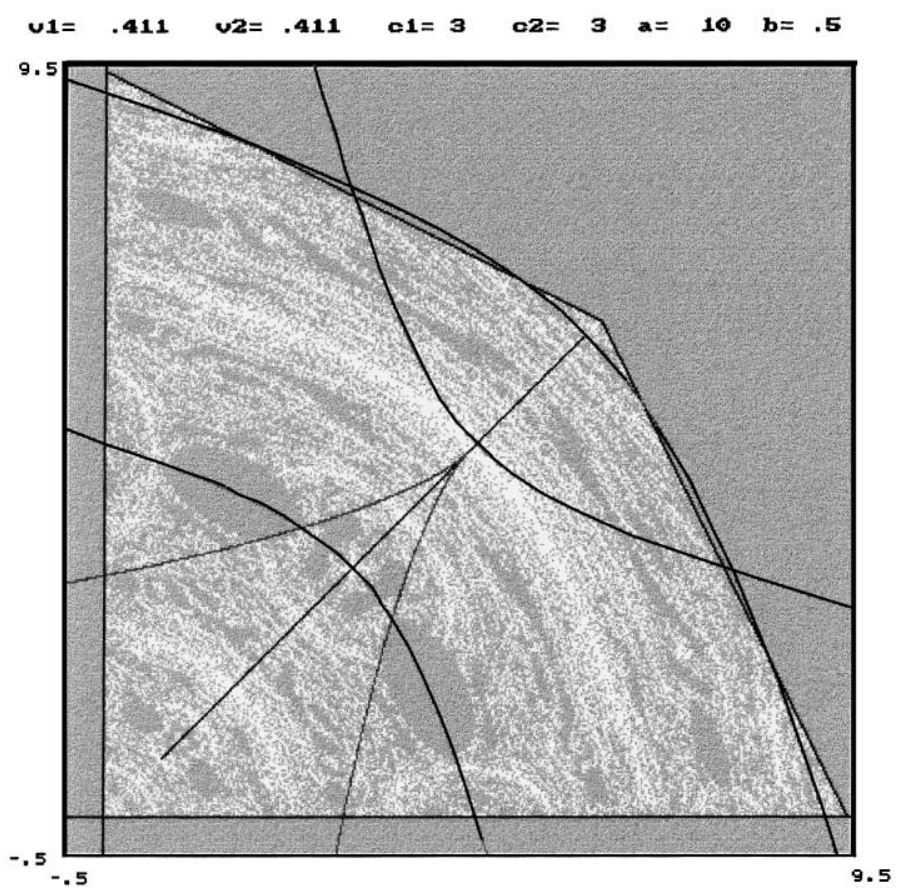

Fig. 8. (a) Expanded version of Fig. 1 with $v$ ranging from 0.410 to 0.415 , in order to show a more detailed structure of the Lyapunov exponent $L_{\perp}$. (b) For $c=3, a=10, b=0.5$ (as in Fig. 8(a)) and $v=0.411$, the numerically obtained basins are shown. For each initial condition, taken over a grid on the portion of phase plane represented in the figure, a trajectory of the map $T_{s}$ has been generated and a grey dot is painted if the phase point reaches the immediate basin of infinity (i.e. the region outside the quadrilateral $O O_{-1}^{(1)} O_{-1}^{(3)} O_{-1}^{(2)}$ ), whereas a white point is painted if after $10^{6}$ iterations the inequality $\left|x_{1}-x_{2}\right|<10^{-7}$ is satisfied. 
many) transversally attracting. For example the fixed point $E_{*}$ is transversally attracting in the whole range of parameters for which bounded attractors exist on $\Delta$.

\subsection{Final bifurcation}

When $v=v_{f}=3 / a-c$, the restriction $\left.T_{s}\right|_{\Delta}$ is conjugated to the standard logistic map $z^{\prime}=\mu z(1-z)$ with $\mu=4$. Hence, for $v>v_{c}$ the generic trajectory starting from a point of the invariant diagonal is divergent. This corresponds also to a global bifurcation for the two-dimensional map. Such bifurcation is called \% final bifurcation in [18,1], where it is defined as the bifurcation given by the contact between a bounded attractor and the boundary of the basin of infinity, causing the destruction of the attractor. In this sense also the contact bifurcation described in Section 4.2 is a final bifurcation at which the invariant area $S$ (which is an attracting set in the usual topological sense) is destroyed. However, another attractor even if in the weak Milnor sense nested inside it survives. Instead at $v=v_{f}$ every bounded attractor also in the weak Milnor sense, disappears

Also this global bifurcation can be characterized by the critical curves. In fact, for $v(a-c)=3$ the critical curve $L C^{(b)}$ passes through $O_{-1}^{(1)}, O_{-1}^{(3)}$ and $O_{-1}^{(2)}$ so that $\mathrm{H}_{0}$ has a contact with the $x_{2}$ axis and the diagonal $\Delta$, in the points $O_{(\overline{2})}^{(2)}$ and $O_{(\overline{3})}^{(3)}$, respectively, and $\mathrm{K}_{0}$ has a contact with the $x_{1}$ axis and the diagonal $\Delta$ in the points $O_{-1}^{(\overline{2})}$ and $O_{-1}^{(\overline{3})}$. Hence their rank-1 preimages, the main holes the $\mathrm{H}_{-1}$ and $\mathrm{K}_{-1}$, have a contact with the same invariant lines in the preimages of $O_{-1}^{(1)}, O_{-1}^{(3)}$ and $O_{-1}^{(2)}$, that in this case are the critical values, located in the critical points of the restrictions $f_{1}, f$ and $f_{2}$, given by $C_{x}=$

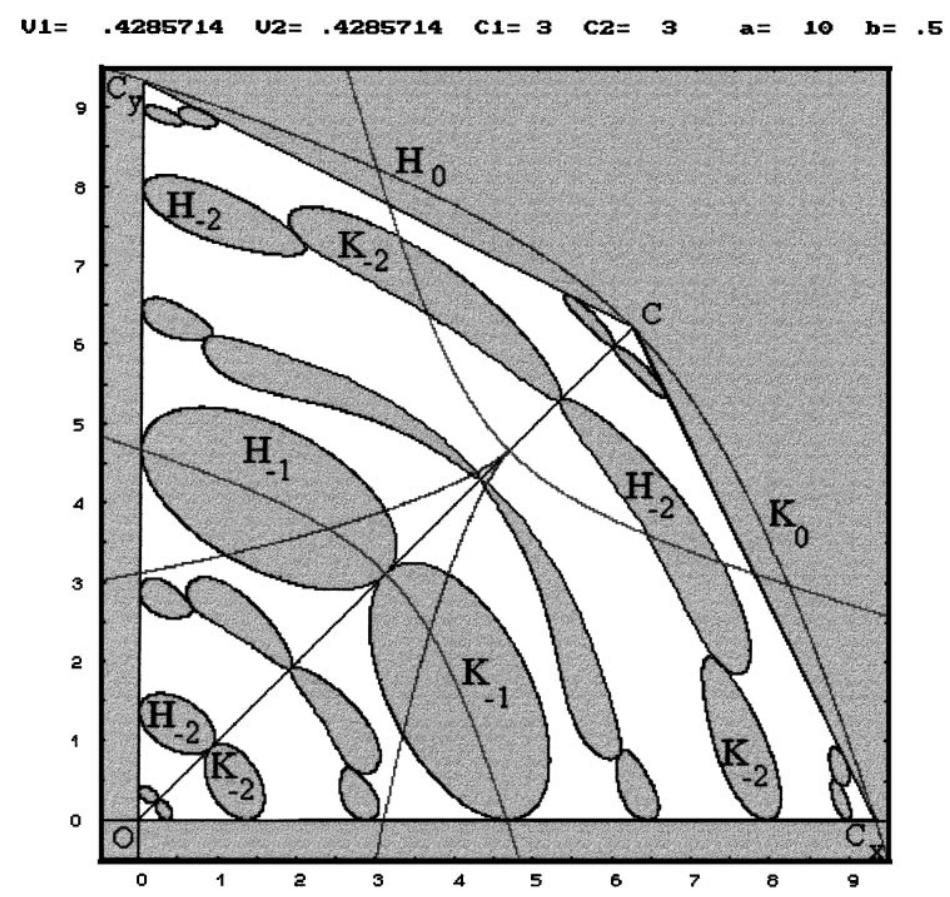

Fig. 9. This is the analogue of Fig. 6, obtained with the same parameters $c, a$ and $b$, but with $v=3 /(a-c)$, at which the final bifurcation occurs. 
$((1+v(a-c)) / 4 b v, 0), C=((1+v(a-c)) / 6 b v,(1+v(a-c)) / 6 b v)$ and $C_{y}=(0,(1+v(a-c)) / 4 b v)$, respectively. Since these three points belong to invariant manifolds, each of them must have at least one of their preimages on the same invariant manifold, so that also the rank-1 preimages of the main holes are tangent to the coordinate axes and to the diagonal. By reasoning inductively it is easy to realize that infinitely many preimages of any rank of the main holes are tangent to these invariant manifolds, the tangency points being the infinite preimages of the critical points of the restrictions $f_{1}, f$ and $f_{2}$ on the respective invariant manifolds. Some of these preimages, up to rank 3, are shown in Fig. 9. For this value of $v(a-c)$, corresponding to the case $\mu=4$ of the standard logistic map (20), the attractor $\mathscr{A}=I_{\Delta}$ is a chaotic interval with an absolutely continuous invariant measure (the logistic map at $\mu=4$ is conjugate to the tent map) and as can be seen in Fig. 1, the transverse natural Lyapunov exponent $L_{\perp}$ is positive. Hence, $\mathscr{A}$ is a chaotic saddle but even if $\mu(\mathscr{B}(\mathscr{A}))=0$, a dense subset of transversally attracting points embedded into $\mathscr{A}$ exists, including the fixed point $E_{*}$ and all its preimages on $\Delta$. In fact, from (26) we can see that $\lambda_{\perp}^{E_{*}}=0$ if $v=3 / a-c$.

We observe that this fact may cause noticeable numerical effects, which may lead to an erroneous interpretation of numerical experiments. In fact it may happen that the attractor $\mathscr{A} \subset \Delta$ is numerically seen even if $\mu(\mathscr{B}(\mathscr{A}))=0$, due to the finite representation of 'real' numbers in computers.

In order to clarify this numerical effect we recall a well known 'numerical paradox'. Suppose, for a one-dimensional map that an invariant set attracts all (and only) the rational numbers then its basin of attraction has one-dimensional Lebesgue measure equal to zero, but in a numerical experiment it seems to attract every trajectory, since only a subset of rational numbers can be represented by a computer.

\section{Conclusions}

In this paper, we have studied phenomena of synchronization, intermittency and the complex structure of the basins of attraction for a two-dimensional noninvertible map with an invariant onedimensional submanifold where synchronized dynamics takes place.

The map describes the time evolution of an economic duopoly system. We have argued that symmetric maps with invariant submanifolds of lower dimensionality naturally arise in the modeling of discrete time dynamic games with identical players. However, these symmetric maps are quite different from those, usually studied in the literature, where there exists a parameter that affects the dynamics transverse to the invariant manifold but does not influence the dynamics on the invariant manifold, i.e. a normal parameter, following the terminology of [4]. In our model each parameter influences both.

The main local and global bifurcations are studied as $v(a-c)$ increases, where $v$ is the speed of adjustment and $(a-c)$ is the difference between maximum unitary price and unitary production cost.

The main results of the paper concern the global behavior of the map out of the invariant manifold where synchronization occurs. Such global behavior is characterized by the properties of the critical curves of the noninvertible map.

Portions of critical curves of increasing rank bound an invariant asymptotically attracting twodimensional set that includes the one-dimensional Milnor attractor to which the synchronized trajectories converge. Such a two-dimensional trapping region gives an upper bound to the intermittency phenomena and becomes the only attractor when the Milnor attractor is transformed into a chaotic saddle. We show that the folding action of the critical curves places an upper bound on how the trajectories starting near the invariant submanifold can get away from it. In other words, the 
study of the critical curves leads to an estimate of the amplitude of the 'bursts' transverse to $\Delta$, i.e. of the maximum difference between the non-synchronized production decisions.

Global bifurcations, that change the structure of the basins of attraction and cause the destruction of the bounded attracting sets, are characterized as contact bifurcations due to tangencies between critical curves and basin boundaries. The effects of such global bifurcations are evidenced both for the changes induced in the basins structure and for the different cases, usually described in the literature, that characterize the possible behaviors of the locally repelled trajectories starting close to the invariant manifold which contains a Milnor attractor which is not asymptotically stable. In fact, trajectories locally repelled by transversally repelling cycles embedded inside the invariant submanifold can be reinjected toward it or can go to another attractor (the infinity in our example) depending on the global properties of the map. The occurrence of contact bifurcations marks the switch from the former to the latter case.

From the point of view of the economic duopoly game, whose dynamic evolution is modeled by the two-dimensional symmetric map (17), the question addressed is that of the asymptotic behavior of two identical producers that enter the economy with different initial productions $x_{1,0} \neq x_{2,0}$. It is plain that identical producers starting with identical initial productions $x_{1,0}=x_{2,0}$ will continue to produce the same quantities for each time $t \geq 0$, i.e. they are synchronized. This implies that their common behavior can be described by the simpler one-dimensional map given by the restriction of the two-dimensional model to the invariant diagonal. But what happens if two identical producers start with different initial productions? Do the endogenous dynamics of the economic system act so that the initial difference is gradually eliminated and synchronization of the productions is reached in the long run, or will the initial difference be enhanced until the duopoly is destroyed (i.e. one producer exits the market)? The results given in this paper show that an answer is not trivial. Asymptotic synchronization occurs for some sets of parameters, so that the simpler one-dimensional map can be used to describe the long-run evolution of the system, but global bifurcations can occur at which intermittent behavior appear, and other bifurcations can give a very complex structure of the basin of attraction of the synchronized dynamics. In this case unbounded trajectories can be obtained (which mean the destruction of the duopoly) even for games starting with quasi identical initial productions.

\section{Acknowledgements}

We are grateful to Professor C. Mira and Y. Maistrenko for very interesting discussions and an anonymous referee for useful comments. The work has been performed under the activity of the national research project 'Dinamiche non lineari ed applicazioni alle scienze economiche e sociali', MURST, Italy, and under the auspices of GNFM, CNR, Italy.

\section{References}

[1] R. Abraham, L. Gardini, C. Mira, Chaos in discrete dynamical systems (a visual introduction in two dimension) Springer-Verlag, 1997.

[2] J.C. Alexander, J.A. Yorke, Z. You, I. Kan, Riddled basins, Int. J. Bif. Chaos 2 (1992) 795-813.

[3] P. Ashwin, J. Buescu, I. Stewart, Bubbling of attractors and synchronization of chaotic oscillators, Phys. Lett. A 193 (1992) 126-139. 
[4] P. Ashwin, J. Buescu, I. Stewart, From attractor to chaotic saddle: a tale of transverse instability, Nonlinearity 9 (1996) 703-737.

[5] G.I. Bischi, A. Naimzada, Global analysis of a dynamic duopoly game with bounded rationality, Annals of Dynamic Games, in press.

[6] G.I. Bischi, M. Gallegati, A. Naimzada, Symmetry-breaking bifurcations and representative firm in dynamic duopoly games, 1997, submitted.

[7] P. Collet, J.P. Eckmann, Iterated maps on the interval as dynamical systems, Birkhäuser, Boston, 1980.

[8] R.L. Devaney, An introduction to chaotic dynamical systems, Benjamin/Cummings, Menlo Park, California, 1987.

[9] A. Ferretti, N.K. Rahman, A study of coupled logistic maps and its applications in chemical physics, Chem. Phys. 119 (1988) 275-288.

[10] L. Gardini, R. Abraham, R. Record, D. Fournier-Prunaret, A double logistic map, Int. J. Bif. Chaos 4(1) (1994) 145-176.

[11] I. Gumowski, C. Mira, Dynamique Chaotique, Cepadues Editions, Toulose, 1980.

[12] T. Hogg, B.A. Huberman, Generic behavior of coupled oscillators, Phys. Rev. A 29 (1984) 275-281.

[13] Y.C. Laj, C. Grebogi, J.A. Yorke, Riddling bifurcation in chaotic dynamical systems, Phys. Rev. Lett. 77 (1996) 55-58.

[14] M. Hasler, Yu. Maistrenko, An introduction to the synchronization of chaotic systems: coupled skew tent maps, 1997, submitted .

[15] Y. Maistrenko, V. Maistrenko, A. Popovich, E. Mosekilde, Transverse instability and riddled basins in a system of two coupled logistic maps, submitted.

[16] J. Milnor, On the concept of attractor, Comm. Math. Phys. 99 (1985) 177-195.

[17] C. Mira, D. Fournier-Prunaret, L. Gardini, H. Kawakami, J.C. Cathala, Basin bifurcations of two-dimensional noninvertible maps: fractalization of basins, Int. J. Bif. Chaos 4(2) (1994) 343-381.

[18] C. Mira, L. Gardini, A. Barugola, J.C. Cathala, Chaotic dynamics in two-dimensional noninvertible maps, World Scientific, 1996.

[19] C. Mira, Chaotic dynamics, World Scientific, 1987.

[20] E. Ott, J.C. Sommerer, Blowout bifurcations: the occurrence of riddled basins, Phys. Lett. A 188 (1994) 39-47.

[21] A. Pikovsky, P Grassberg, Symmetry breaking bifurcation for coupled chaotic attractors., J. Phys. A: Math Gen. 24 (1991) 4587-4597.

[22] C. Reick, E. Mosekilde, Emergence of quasiperiodicity in symmetrical coupled, identical period-doubling systems, Phys. Rev. E 52 (1995) 1428-1434.

[23] A.N. Sharkovsky, Yu.L. Maistrenko, E.Yu. Romanenko, Difference equations and their applications, Kluwer Academic Publishers, 1993.

[24] S.C. Venkataramani, B.R. Hunt, E. Ott, D.J. Gauthier, J.C. Bienfang, Transition to bubbling of chaotic systems, Phys. Rev. Letts. 77 (1996) 5361-5364.

[25] S.C. Venkataramani, B.R. Hunt, E. Ott, Bubbling transition, Phys. Rev. E 54 (1996) 1346-1360. 